\title{
Shaping Pulses to Control Bistable Systems: Analysis, Computation and Counterexamples ${ }^{1}$
}

\author{
Aivar Sootla $^{\mathrm{a}}$, Diego Oyarzún ${ }^{\mathrm{b}}$, David Angeli ${ }^{\mathrm{c}}$ and Guy-Bart Stan ${ }^{\mathrm{d}}$, \\ ${ }^{\mathrm{a}}$ Montefiore Institute, University of Liège, B-4000, Belgium \\ ${ }^{\mathrm{b}}$ Department of Mathematics, Imperial College London, UK \\ ${ }^{\mathrm{c}}$ Department of Electrical and Electronic Engineering, Imperial College London, UK and Department of Information Engineering, \\ University of Florence, Italy \\ ${ }^{\mathrm{d}}$ Department of Bioengineering, Imperial College London, UK
}

\begin{abstract}
In this paper we study how to shape temporal pulses to switch a bistable system between its stable steady states. Our motivation for pulse-based control comes from applications in synthetic biology, where it is generally difficult to implement real-time feedback control systems due to technical limitations in sensors and actuators. We show that for monotone bistable systems, the estimation of the set of all pulses that switch the system reduces to the computation of one non-increasing curve. We provide an efficient algorithm to compute this curve and illustrate the results with a genetic bistable system commonly used in synthetic biology. We also extend these results to models with parametric uncertainty and provide a number of examples and counterexamples that demonstrate the power and limitations of the current theory. In order to show the full potential of the framework, we consider the problem of inducing oscillations in a monotone biochemical system using a combination of temporal pulses and event-based control. Our results provide an insight into the dynamics of bistable systems under external inputs and open up numerous directions for future investigation.
\end{abstract}

\section{Introduction}

In this paper we investigate how to switch a bistable system between its two stable steady states using external input signals. Our main motivation for this problem comes from synthetic biology, which aims to engineer and control biological functions in living cells [3]. Most of current research in synthetic biology focuses on building biomolecular circuits inside cells through genetic engineering. Such circuits can control cellular functions and implement new ones, including cellular logic gates, cell-to-cell communication and light-responsive behaviours. These systems have enormous

\footnotetext{
Email addresses: asootlaeulg.ac.be (Aivar Sootla), d.oyarzun@imperial.ac.uk (Diego Oyarzún), doctoral research associate at ICL. Dr Sootla and Dr Stan acknowledge support by the EPSRC grant EP/G036004/1. Dr Stan is additionally supported by the EPRSC grants EP/J014214/1, EP/M002187/1. Dr Sootla is now supported by an F.R.S.-FNRS Fellowship. Dr Oyarzún is supported by a Junior Research Fellowship from ICL. The authors would like to thank Dr Alexandre Mauroy for valuable suggestions and discussions.
}

potential in diverse applications such as metabolic engineering, bioremediation, and even the energy sector [18].

Several recent works $[16,13,29]$ have showcased how cells can be controlled externally via computer-based feedback and actuators such as chemical inducers or light stimuli $[14,12]$. An important challenge in these approaches is the need for real-time measurements, which tend to be costly and difficult to implement with current technologies. In addition, because of technical limitations and the inherent nonlinearity of biochemical interactions, actuators are severely constrained in the type of input signals they can produce. As a consequence, the input signals generated by traditional feedback controllers (e.g. PID or model predictive control) may be hard to implement without a significant decrease in control performance.

In this paper we show how to switch a bistable system without the need for output measurements. We propose an openloop control strategy based on a temporal pulse of suitable magnitude $\mu$ and duration $\tau$ :

$$
u(t)=\mu h(t, \tau), \quad h(t, \tau)= \begin{cases}1 & 0 \leq t \leq \tau \\ 0 & t>\tau\end{cases}
$$


Our goal is to characterise the set of all pairs $(\mu, \tau)$ that can switch the system between the stable steady states and the set of all pairs $(\mu, \tau)$ that cannot. We call these sets the switching sets and a boundary between these sets the switching separatrix. The pairs $(\mu, \tau)$ close to the switching separatix are especially important in synthetic biology applications, as a large $\mu$ or a large $\tau$ can trigger toxic effects that slow down cell growth or cause cell death.

In a previous paper [23], we showed that for monotone systems the switching separatrix is a monotone curve. This result was therein extended to a class of non-monotone systems whose vector fields can be bounded by vector fields of monotone systems. This idea ultimately leads to robustness guarantees under parametric uncertainty. These results are in the spirit of $[8,19]$, where the authors considered the problem of computing reachability sets of a monotone system. Some parallels can be also drawn with $[15,5]$, where feedback controllers for monotone systems were proposed.

Contributions. In the present paper we provide the first complete proof of our preliminary results in [23] and extend them in several directions. We formulate necessary and sufficient conditions for the existence of the monotone switching separatrix for non-monotone systems. Although it is generally hard to use this result to establish monotonicity of the switching separatrix, we use it to prove the converse. For example, we show that for a bistable Lorenz system the switching separatrix is not monotone. We then generalise the main result of [23] by providing conditions for the switching separatrix to be a graph of a function. We also discuss the relation between bifurcations and the mechanism of the pulse-based switching, which provides additional insights into the switching problem. We use this intuition to show and then explain the failure of pulse-based control on an HIV viral load control problem [1]. We proceed by providing a numerical algorithm to compute the switching separatrices for monotone systems. The algorithm can be efficiently distributed among several computational units and does not explicitly use the vector field of the model. We evaluate the theory and computational tools on the bistable LacI-TetR system, which is commonly referred to as a $g e$ netic toggle switch [7]. Genetic toggle switches are widely used in synthetic biology to trigger cellular functions in response to extracellular signals [3,10].

We complement our theoretical findings with several observations that illustrate the limitations of the current theory and highlight the need for deeper investigations of bistable systems. For example, we show that for a toxin-antitoxin system [4], the switching separatrix appears to be monotone, even though the system does not appear to be monotone. Finally, in order to demonstrate the full potential of pulsebased control, we consider a problem of inducing an oscillatory behaviour in the generalised repressilator system [26].

Organisation. The rest of the paper is organised as follows. In Section 2 we cover the basics of monotone systems theory, formulate the problem in Subsection 2.1, and provide an intuition into the mechanism of the pulse-based switching for monotone systems in Subsection 2.2. We also provide some motivational examples for the development of our theoretical results. In Section 3 we formulate the theoretical results and in Section 4 we present the computational algorithm, which we evaluate in Section 5 on the LacI-TetR system. In Section 6, we provide counterexamples and an application of inducing oscillations in a generalised repressilator system. All the proofs are found in Appendix.

Notation. Let $\|\cdot\|_{2}$ stand for the Euclidean norm in $\mathbb{R}^{n}, Y^{*}$ stand for a topological dual to $Y, X \backslash Y$ stand for the relative complement of $X$ in $Y$, int $(Y)$ stand for the interior of the set $Y$, and $\operatorname{cl}(Y)$ for its closure.

\section{Preliminaries}

Consider single input control systems

$$
\dot{x}=f(x, u), \quad x(0)=x_{0},
$$

where $f: \mathcal{D} \times \mathcal{U} \rightarrow \mathbb{R}^{n}, u: \mathbb{R}_{>0} \rightarrow \mathcal{U}, \mathcal{D} \subset \mathbb{R}^{n}, \mathcal{U} \subset \mathbb{R}$ and $u(\cdot)$ belongs to the space $\mathcal{U}_{\infty}$ of Lebesgue measurable functions with values from $\mathcal{U}$. We say that the system is unforced, if $u=0$. We define the flow map $\phi_{f}: \mathbb{R} \times$ $\mathcal{D} \times \mathcal{U}_{\infty} \rightarrow \mathbb{R}^{n}$, where $\phi_{f}\left(t, x_{0}, u\right)$ is a solution to the system (2) with an initial condition $x_{0}$ and a control signal $u$. We consider the control signals in the shape of a pulse, that is signals defined in (1) with the set of admissible $\mu$ and $\tau$ denoted as $\mathcal{S}=\left\{\mu, \tau \in \mathbb{R}_{\geq 0}\right\}$.

In order to avoid confusion, we reserve the notation $f(x, u)$ for the vector field of non-monotone systems, while systems

$$
\begin{array}{ll}
\dot{x}=g(x, u), & x(0)=x_{0}, \\
\dot{x}=r(x, u), & x(0)=x_{0},
\end{array}
$$

denote so-called monotone systems throughout the paper. In short, monotone systems are those which preserve a partial order relation in initial conditions and input signals. A relation $\sim$ is called a partial order if it is reflexive $(x \sim x)$, transitive $(x \sim y, y \sim z$ implies $x \sim z)$, and antisymmetric $(x \sim y, y \sim x$ implies $x=y$ ). Partial orders also induce some geometric properties on sets. A set $M$ is called $p$-convex if for every $x, y$ in $M$ such that $x \succeq_{x} y$, and every $\lambda \in(0,1)$ we have that $\lambda x+(1-\lambda) y \in M$. We define a partial order $\succeq_{x}$ through a cone $K \in \mathbb{R}^{n}$ as follows: $x \succeq_{x} y$ if and only if $x-y \in K$. We write $x \succeq_{x} y$, if the relation $x \succeq_{x} y$ does not hold. We will also write $x \succ_{x} y$ if $x \succeq_{x} y$ and $x \neq y$, and $x \gg_{x} y$ if $x-y \in \operatorname{int}(K)$. Similarly we can define a partial order on the space of signals $u \in \mathcal{U}_{\infty}$ : $u \succeq_{u} v$ if $u(t)-v(t) \in K$ for all $t \geq 0$. We write $u \succ_{u} v$, if $u \succeq_{u} v$ and $u(t) \neq v(t)$ for all $t \geq 0$.

Definition 1 The system (3) is called monotone on $\mathcal{D}_{M} \times$ $\mathcal{U}_{\infty}$ with respect to the partial orders $\succeq_{x}$, $\succeq_{u}$, if for all $x, y \in \mathcal{D}_{M}$ and $u, v \in \mathcal{U}_{\infty}$ such that $x \succeq_{x} y$ and $u \succeq_{u} v$, we 
have $\phi_{g}(t, x, u) \succeq_{x} \phi_{g}(t, y, v)$ for all $t \geq 0$. If additionally, $x \succ_{x} y$, or $u \succ_{x} v$ implies that $\phi_{g}(t, x, u) \gg_{x} \phi_{g}(t, y, v)$ for all $t>0$, then the system is called strongly monotone.

In general, it is hard to establish monotonicity of a system with respect to an order other than an order induced by an orthant (e.g., positive orthant $\mathbb{R}_{\geq 0}^{n}$ ). Hence throughout the paper, by a monotone system we actually mean a monotone system with respect to a partial order induced by an orthant. A certificate for monotonicity with respect to an orthant is referred to as Kamke-Müller conditions [2].

Proposition 2 ([2]) Consider the system (3), where $g$ is differentiable in $x$ and $u$ and let the sets $\mathcal{D}_{M}, \mathcal{U}$ be p-convex. Let the partial orders $\succeq_{x}, \succeq_{u}$ be induced by $P_{x} \mathbb{R}_{\geq 0}^{n}, P_{u} \mathbb{R}_{\geq 0}^{m}$, respectively, where $P_{x}=\operatorname{diag}\left((-1)^{\varepsilon_{1}}, \ldots,(-1)^{\varepsilon_{n}}\right), P_{u}=$ $\operatorname{diag}\left((-1)^{\delta_{1}}, \ldots,(-1)^{\delta_{m}}\right)$ for some $\varepsilon_{i}, \delta_{i}$ in $\{0,1\}$. Then

$$
\begin{gathered}
(-1)^{\varepsilon_{i}+\varepsilon_{j}} \frac{\partial g_{i}}{\partial x_{j}} \geq 0, \quad \forall i \neq j, \quad(x, u) \in \operatorname{cl}\left(\mathcal{D}_{M}\right) \times \mathcal{U} \\
(-1)^{\varepsilon_{i}+\delta_{j}} \frac{\partial g_{i}}{\partial u_{j}} \geq 0, \quad \forall i, j, \quad(x, u) \in \mathcal{D}_{M} \times \mathcal{U}
\end{gathered}
$$

if and only if the system (3) is monotone on $\mathcal{D}_{M} \times \mathcal{U}_{\infty}$ with respect to $\succeq_{x}, \succeq_{u}$

If we consider the orthants $\mathbb{R}_{\geq 0}^{n}, \mathbb{R}_{>0}^{m}$, then the conditions above are equivalent to checking if for all $x \preceq_{x} y$ such that $x_{i}=y_{i}$ for some $i$, and all $u \preceq_{u} v$ we have $g_{i}(x, u) \leq$ $g_{i}(y, v)$.

\subsection{Problem Formulation}

We confine the class of considered control systems by making the following assumptions:

A1. Let $f(x, u)$ in (2) be continuous in $(x, u)$ on $\mathcal{D}_{f} \times \mathcal{U}$. Moreover, for each compact sets $C_{1} \subset \mathcal{D}_{f}$ and $C_{2} \subset$ $\mathcal{U}$, let there exist a constant $k$ such that $\| f(\xi, u)-$ $f(\zeta, u)\left\|_{2} \leq k\right\| \xi-\zeta \|_{2}$ for all $\xi, \zeta \in C_{1}$ and $u \in C_{2}$.

A2. Let the unforced system (2) have two stable steady states in $\mathcal{D}_{f}$, denoted as $s_{f}^{0}$ and $s_{f}^{1}$,

A3. Let $\mathcal{D}_{f}=\operatorname{cl}\left(\mathcal{A}\left(s_{f}^{0}\right) \cup \mathcal{A}\left(s_{f}^{1}\right)\right)$, where $\mathcal{A}\left(s_{f}^{i}\right)$ stands for the domain of attraction of the steady state $s_{f}^{i}$ for $i=0,1$ of the unforced system (2),

A4. For any $u=\mu h(\cdot, \tau)$ with finite $\mu$ and $\tau$ let $\phi_{f}\left(t, s_{f}^{0}, u\right)$ belong to $\operatorname{int}\left(\mathcal{D}_{f}\right)$. Moreover, let the sets

$$
\begin{aligned}
& \mathcal{S}_{f}^{+}=\left\{\mu, \tau>0 \mid \lim _{t \rightarrow \infty} \phi_{f}\left(t ; s_{f}^{0}, \mu h(\cdot, \tau)\right)=s_{f}^{1}\right\} \\
& \mathcal{S}_{f}^{-}=\left\{\mu, \tau>0 \mid \lim _{t \rightarrow \infty} \phi_{f}\left(t ; s_{f}^{0}, \mu h(\cdot, \tau)\right)=s_{f}^{0}\right\}
\end{aligned}
$$

be non-empty.

Assumption A1 guarantees existence, uniqueness and continuity of solutions to (2), while Assumptions A2-A3 define a bistable system on a set $\mathcal{D}_{f}$ controlled by pulses. In

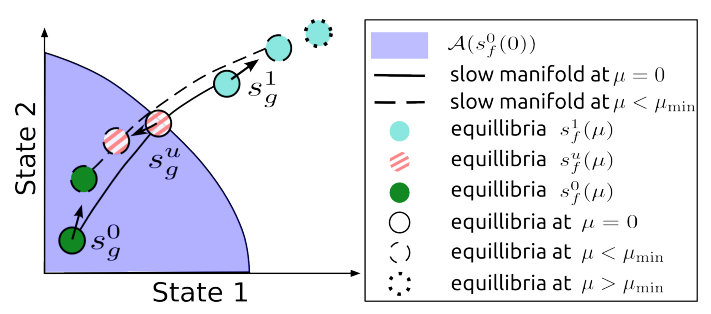

Fig. 1. A schematic depiction of the evolution of the stable nodes $s_{g}^{0}(\mu), s_{g}^{1}(\mu)$ and the saddle $s_{g}^{u}(\mu)$ with respect to $\mu$ in the genetic toggle switch system. By slow manifold we mean a manifold connecting stable equillibria and a saddle. The arrows show the direction of the equillibria movements with increasing $\mu$. At $\mu_{\min }$ the equilibria $s_{g}^{0}(\mu)$ and $s_{g}^{u}(\mu)$ collide resulting in a saddle-node bifurcation preserving only $s_{g}^{1}(\mu)$.

Assumption A4 we define the switching sets: the set $\mathcal{S}_{f}^{+}$, which contains all $(\mu, \tau)$ pairs that switch the system, and the set $\mathcal{S}_{f}^{-}$, which contains all pairs that do not. The boundary between these sets is called the switching separatrix. In the rest of the paper, we focus on the control problem of estimating the switching sets.

\subsection{Mechanism of Pulse-Based Switching}

The general problem of switching a bistable system with external inputs is amenable to an optimal control formulation. However, in applications such as synthetic biology, optimal control solutions can be very hard to implement due to technical limitations in actuators and output measurements. Additionally, the solution of this optimal control problem may be technically challenging. Hence applying open-loop pulses can be a reasonable solution, if we can guarantee some form of robustness. As we shall see later, our results show that for monotone systems, pulse-based switching is computationally tractable and robust towards parameter variations.

Before presenting our main results, we first provide an intuitive link between monotonicity and the ability to switch a system with temporal pulses. If we consider constant inputs $u=\mu$ and regard $\mu$ as a bifurcation parameter, we have the following result.

Proposition 3 Let the system (3) satisfy Assumptions A1-A4 and be monotone on $\mathcal{D}_{g} \times \mathcal{U}_{\infty}$ with respect to $\mathbb{R}_{\geq 0}^{n}, \mathbb{R}_{\geq 0}$. Let $\mu_{\min }$ be such that all pairs $(\mu, \tau) \in \mathcal{S}_{g}^{-}$for $0<\mu<\mu_{\min }$, and any finite positive $\tau$. Let also $\xi(\mu)=\lim _{t \rightarrow \infty} \phi_{g}\left(t, s_{g}^{0}, \mu\right)$ and $\eta(\mu)=\lim _{t \rightarrow \infty} \phi_{g}\left(t, s_{g}^{1}, \mu\right)$. Then

(1) If $\mu \leq \lambda<\mu_{\min }$ then $\xi(\mu) \preceq_{x} \xi(\lambda), \eta(\mu) \preceq_{x} \eta(\lambda)$;

(2) If $0<\mu<\mu_{\min }$ then $\xi(\mu) \in \mathcal{A}\left(s_{g}^{0}\right)$ and $\xi(\mu) \prec_{x} \eta(\mu)$;

(3) The function $\xi(\mu)$ is discontinuous at $\mu_{\mathrm{min}}$.

The proof of the proposition is in Appendix. In many applications, the functions $\xi(\mu), \eta(\mu)$ are simply evolutions of the steady states $s_{g}^{0}, s_{g}^{1}$ with respect to the parameter $\mu$, respectively. Hence, statement (1) of Proposition 3 shows how the 
steady states move with respect to changes in $\mu$. Statement (2) ensures that there are at least two distinct asymptotically stable equilibria for $\mu<\mu_{\min }$. Finally, statement (3) indicates that the system undergoes a bifurcation for $\mu=\mu_{\min }$, as illustrated in Figure 1. The particular type of the bifurcation will depend on a specific model. Next we investigate further aspects of this result with some examples of monotone and non-monotone bistable systems.

Example 1: LacI-TetR Switch. The genetic toggle switch is composed of two mutually repressive genes LacI and TetR and was a pioneering genetic system for synthetic biology [7]. We consider its control-affine model, which is consistent with a toggle switch actuated by light induction [12]:

$$
\begin{aligned}
& \dot{x}_{1}=\frac{p_{1}}{1+\left(x_{2} / p_{2}\right)^{p_{3}}}+p_{4}-p_{5} x_{1}+u, \\
& \dot{x}_{2}=\frac{p_{6}}{1+\left(x_{1} / p_{7}\right)^{p_{8}}}+p_{9}-p_{10} x_{2},
\end{aligned}
$$

where the parameters have the following values

$$
\begin{aligned}
& p_{1}=40, \quad p_{2}=1, \quad p_{3}=4, \quad p_{4}=0.05, \quad p_{5}=1, \\
& p_{6}=30, \quad p_{7}=1, \quad p_{8}=4, \quad p_{9}=0.1, \quad p_{10}=1 \text {. }
\end{aligned}
$$

In the model (5), $x_{i}$ represents the concentration of each protein, whose mutual repression is modelled via a rational function. The parameters $p_{2}$ and $p_{7}$ represent repression thresholds, whereas $p_{4}$ and $p_{9}$ model the basal synthesis rate of each protein. The parameters $p_{5}$ and $p_{10}$ are degradation rate constants, and $p_{1}, p_{6}$ describe the strength of mutual repression. By means of Proposition 2 we can readily check that the model is monotone on $\mathbb{R}_{\geq 0}^{2} \times \mathbb{R}_{\geq 0}$ for all nonnegative parameter values. It can be verified by direct computation that the system satisfies Assumptions A1-A4 with $\mathcal{D}_{f}=\mathbb{R}_{\geq 0}^{2}$. It can be also shown that the unforced system is strongly monotone in $\operatorname{int}\left(\mathbb{R}_{>0}^{2}\right)$ using the results in [20].

With the chosen parameter values, we numerically found a bifurcation to occur at $\mu_{\min } \approx 1.4077$. For $\mu<\mu_{\min }$ the system has two stable nodes and a saddle. We observe that $\xi(\mu)=\eta(\mu)$ for all $\mu>\mu_{\min }$, and therefore we conclude that the system undergoes a saddle-node bifurcation.

\section{Example 2: Lorenz system. Consider a system}

$$
\begin{aligned}
& \dot{x}_{1}=\sigma\left(x_{2}-x_{1}\right)+u \\
& \dot{x}_{2}=x_{1}\left(\rho-x_{3}\right)-x_{2}+u \\
& \dot{x}_{3}=x_{1} x_{2}-\beta x_{3}
\end{aligned}
$$

with parameters $\sigma=10, \rho=21, \beta=8 / 3$, which is nonmonotone and bistable with two stable foci. Numerical computation of the sets $\mathcal{S}^{-}$and $\mathcal{S}^{+}$in Figure 2 suggests that the switching separatrix is not monotone. We will revisit this conclusion in the next section using our theoretical results.

Example 3: HIV viral load control problem. In [1] the authors considered the problem of switching from "non-healthy"

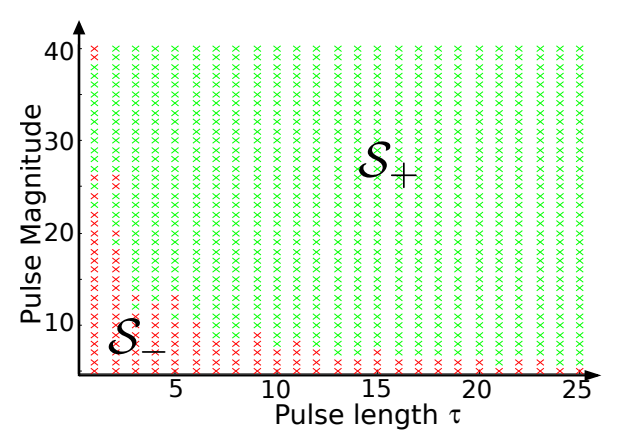

Fig. 2. Switching sets for the Lorenz system. We simulated the Lorenz system for $(\mu, \tau)$ pairs taken from a mesh grid. The green and red crosses correspond to the pairs that switched or not switched the system, respectively.

$\left(s^{0}\right)$ to a "healthy" $\left(s^{1}\right)$ steady state by means of two control inputs $\left(u_{1}\right.$ and $\left.u_{2}\right)$ that model different drug therapies. Due to space limitations we refer the reader to [1] for a description of the model. It can be shown that both steady states are stable foci and that the model is non-monotone. Although the system can be switched with non-pulse control signals [1], using extensive simulations we were unable to find a combination of pulses in $u_{1}$ and $u_{2}$ switching the system.

As in the case of a monotone bistable system, we found a bifurcation with respect to constant control signals $u_{1}=\mu_{1}$ and $u_{2}=\mu_{2}$. More specifically, we fixed $\mu_{2}=0.4$, and numerically found a bifurcation at $\mu_{1} \approx 0.7059$. The major difference between this case and the monotone system case (Example 1) is that the steady state $s^{1}(0.7059,0.4)$ lies the domain of attraction of $s^{0}(0,0)$. Hence if we stop applying the constant control signal we regress back to the initial point $s^{0}(0,0)$. Furthermore, with increasing $\mu_{1}$ the steady state $s^{1}\left(\mu_{1}, 0.4\right)$ is moving towards the origin, which also lies in the domain of attraction of $s^{0}(0,0)$. This makes pulse-based switching very difficult, if not impossible.

\section{Theoretical Results}

In [23] we showed that the switching separatrix of a monotone bistable system $\dot{x}=g(x, u)$ is non-increasing. Here we present a generalisation of this result by formulating necessary and sufficient conditions for the switching separatrix to be monotone, the proof of which is found in Appendix.

Theorem 4 Let the system (2) satisfy Assumptions A1-A4. Then the following properties are equivalent:

(1) If $\phi_{f}\left(t, s_{f}^{0}, \bar{\mu} h(\cdot, \bar{\tau})\right)$ belongs to $\mathcal{A}\left(s_{f}^{0}\right)$ for all $t>0$, then $\phi_{f}\left(t, s_{f}^{0}, \mu h(\cdot, \tau)\right)$ belongs to $\mathcal{A}\left(s_{f}^{0}\right)$ for all $t \geq 0$, and for all $\mu, \tau$ such that $0<\mu \leq \bar{\mu}, 0<\tau \leq \bar{\tau}$.

(2) The set $\mathcal{S}_{f}^{-}$is simply connected. There exists a curve $\mu_{f}(\tau)$, which is a set of maximal elements of $\mathcal{S}_{f}^{-}$in the standard partial order. Moreover, the curve $\mu_{f}(\tau)$ is such that for any $\mu_{1} \in \mu_{f}\left(\tau_{1}\right)$ and $\mu_{2} \in \mu_{f}\left(\tau_{2}\right)$, $\mu_{1} \geq \mu_{2}$ for $\tau_{1}<\tau_{2}$. 
Theorem 4 shows that the computation of the set $\mathcal{S}_{f}^{-}$is reduced to the computation of a curve $\mu_{f}(\tau)$. This result also provides a connection between the geometry of domains of attraction of the unforced system and the switching separatrix. As shown next, Theorem 4 can also be used to establish the non-monotonicity of the switching separatrix.

Remark 5 (Lorenz system revisited) Consider the Lorenz system from the previous section and three different pulses $u_{i}(t)=\mu_{i} h(t, \tau)$ with $\mu_{1}=24, \mu_{2}=25, \mu_{3}=26$, and $\tau=1$. Numerical solutions with increased accuracy show that the flows $\phi\left(t, s^{0}, u_{1}\right)$ and $\phi\left(t, s^{0}, u_{3}\right)$ converge to $s^{0}$, whereas $\phi\left(t, s^{0}, u_{2}\right)$ converges to $s^{1}$. Application of Theorem 4 proves that the switching separatrix is not monotone.

The major bottleneck in the direct application of Theorem 4 is the verification of condition (1), which is generally computationally intractable. For example, condition (1) is satisfied if the partial order is preserved for control signals. That is for any $u \preceq_{u} v$, it should follow that $\phi_{g}\left(t, s_{g}^{0}, u\right) \preceq_{x} \phi_{g}\left(t, s_{g}^{0}, v\right)$ for all $t>0$. Although this property is weaker than monotonicity, it is not clear how to verify it. Monotonicity, on the other hand, is easy to check and implies condition (1) in Theorem 4. This is used in the following result.

Theorem 6 Let the system (3) satisfy Assumptions A1-A4 and be monotone on $\mathcal{D}_{g} \times \mathcal{U}_{\infty}$.

(1) The set $\mathcal{S}_{g}^{-}$is simply connected. There exists a curve $\mu_{g}(\tau)$, which is a set of maximal elements of $\mathcal{S}_{g}^{-}$in the standard partial order. Moreover, the curve $\mu_{g}(\tau)$ is such that for any $\mu_{1} \in \mu_{g}\left(\tau_{1}\right)$ and $\mu_{2} \in \mu_{g}\left(\tau_{2}\right)$, $\mu_{1} \geq \mu_{2}$ for $\tau_{1}<\tau_{2}$.

(2) The set $\mathcal{S}_{g}^{+}$is simply connected. There exists a curve $\nu_{g}(\tau)$, which is a set of minimal elements of $\mathcal{S}_{g}^{+}$in the standard partial order. Moreover, the curve $\nu_{g}(\tau)$ is such that for any $\nu_{1} \in \nu_{g}\left(\tau_{1}\right)$ and $\nu_{2} \in \nu_{g}\left(\tau_{2}\right)$, $\nu_{1} \geq \nu_{2}$ for $\tau_{1}<\tau_{2}$.

(3) Let the system (3) be strongly monotone and $\partial \mathcal{A}$ be the separatrix between the domains of attractions $\mathcal{A}\left(s_{f}^{0}\right)$ and $\mathcal{A}\left(s_{f}^{1}\right)$ of the unforced system (3). Let additionally $\partial \mathcal{A}$ be an unordered manifold, that is, there are no $x$, $y$ in $\partial \mathcal{A}$ such that $x \succ_{x} y$. Then $\nu_{g}(\tau)=\mu_{g}(\tau)$ for all $\tau>0$ and the curve $\mu_{g}(\cdot)=\nu_{g}(\cdot)$ is a graph of a monotonically decreasing function.

We state implicitly in Theorem 6, that if $\mu_{g}(\cdot) \neq \nu_{g}(\cdot)$, then the flow $\phi_{g}\left(t, s_{g}^{0}, \mu h(\cdot, \tau)\right)$ does not converge to $s_{g}^{0}$ or $s_{g}^{1}$, since it may end up on the separatrix $\partial \mathcal{A}$. We note that our computational procedure presented in Section 4 does not require that $\mu_{g}(\tau)=\nu_{g}(\tau)$ or that $\mu_{g}(\cdot), \nu_{g}(\cdot)$ are graphs of functions. Hence we treat point (3) in Theorem 6 as a strictly theoretical result, but remark that sufficient conditions for the separatrix $\partial \mathcal{A}$ to be unordered are provided in Theorem 2.1 in [9]. The most relevant condition to our case is that the flow of the unforced system is strongly monotone, which we also assume in Theorem 6 .
Besides the separatrix $\partial \mathcal{A}$ being an unordered set, there are other pathological cases. For example, applying constant input control signals $u=\mu$ typically results in a system (2) with a different set of steady states than $s_{f}^{0}$ or $s_{f}^{1}$. Moreover, the number of equilibria may be different. Hence, with $\tau \rightarrow \infty$ the set $\mathcal{S}_{f}^{+}$typically does not contain the limiting control signal $u=\mu$. If the set of pairs $(\mu, \tau)$ resulting in these pathological cases is not measure zero, then the sets $\operatorname{cl}\left(\mathcal{S}_{f}^{+}\right)$and $\operatorname{cl}\left(\mathbb{R}_{\geq 0}^{2} \backslash \mathcal{S}_{f}^{-}\right)$are not equal, which can complicate the computation of the switching sets. However, in many practical applications, the sets $\operatorname{cl}\left(\mathcal{S}_{f}^{+}\right)$and $\operatorname{cl}\left(\mathbb{R}_{\geq 0}^{2} \backslash \mathcal{S}_{f}^{-}\right)$appear to be equal. Therefore in order to simplify the presentation we study only the properties of the set $\mathcal{S}_{f}^{-}$.

If the system $\dot{x}=f(x, u)$ to be controlled is not monotone, then the curve $\mu_{f}(\tau)$ may not be monotone, which is essential for our computational procedure. Instead, we estimate inner and outer bounds on the switching set provided that the vector field of the system can be bounded from above and below by vector fields of monotone systems. This is formally stated in the next result, while the proof is in Appendix.

Theorem 7 Let systems (2), (3), (4) satisfy Assumptions A1A4. Let $\mathcal{D}_{M}=\mathcal{D}_{g} \cup \mathcal{D}_{f} \cup \mathcal{D}_{r}$, the systems (3) and (4) be monotone on $\mathcal{D}_{M} \times \mathcal{U}_{\infty}$ and

$$
g(x, u) \preceq_{x} f(x, u) \preceq_{x} r(x, u) \text { on } \mathcal{D}_{M} \times \mathcal{U}
$$

Assume that the stable steady states $s_{g}^{0}, s_{f}^{0}, s_{r}^{0}, s_{f}^{1}$ satisfy

$$
\begin{gathered}
s_{g}^{0}, s_{f}^{0}, s_{r}^{0} \in \operatorname{int}\left(\mathcal{A}\left(s_{g}^{0}\right) \cap \mathcal{A}\left(s_{f}^{0}\right) \cap \mathcal{A}\left(s_{r}^{0}\right)\right), \\
s_{f}^{1} \notin\left\{z \mid s_{g}^{0} \preceq_{x} z \preceq_{x} s_{r}^{0}\right\} .
\end{gathered}
$$

Then the following relations hold:

$$
\mathcal{S}_{g}^{-} \supseteq \mathcal{S}_{f}^{-} \supseteq \mathcal{S}_{r}^{-}
$$

The technical conditions in (8), (9) are crucial to the proof and are generally easy to satisfy. An illustration of these conditions is provided in Figure 3. Checking the condition (9) reduces to the computation of the stable steady states, as does checking the condition (8). Indeed, to verify that $s_{f}^{0}$ belongs to the intersection of $\mathcal{A}\left(s_{g}^{0}\right), \mathcal{A}\left(s_{f}^{0}\right), \mathcal{A}\left(s_{r}^{0}\right)$, we check if the trajectories of the systems (3), (4) initialised at $s_{f}^{0}$ with $u=0$ converge to $s_{g}^{0}$ and $s_{r}^{0}$, respectively, which is done by numerical integration. The computation of stable steady states can be done using the methods from [32].

In some applications, we need to find a subset of the pairs $(\mu, \tau)$ that switch the system (2) from $s_{f}^{0}$ to $s_{f}^{1}$. Due to the inclusion $\mathcal{S}_{g}^{-} \supseteq \mathcal{S}_{f}^{-}$, existence of the system (3) allows to do that. In this case, we are only interested in finding the system (3), hence the condition (9) is not required and the condition (8) is transformed to $s_{g}^{0}, s_{f}^{0} \in \operatorname{int}\left(\mathcal{A}\left(s_{g}^{0}\right) \cap \mathcal{A}\left(s_{f}^{0}\right)\right)$. 


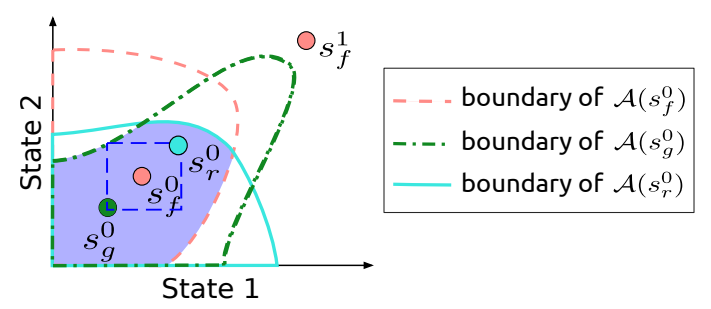

Fig. 3. A schematic depiction of the conditions (8) and (9). The condition (8) ensures that all the steady states lie in the intersection of the corresponding regions of attractions (violet area). The steady state $s_{f}^{1}$ cannot lie in the dashed blue box due to condition (9).

Remark 8 The proofs of Theorems 6 and 7 are adapted in a straightforward manner to the case when systems are monotone with respect to an order $\succeq_{x}$ induced by an arbitrary cone $K_{x}$, and the order $\succeq_{u}$ induced by $\mathbb{R}_{\geq 0}$. In examples, however, we always assume that $K_{x}$ is an orthant.

Theorem 7 also provides a way of estimating the switching set under parametric uncertainty, which is stated in the next corollary.

Corollary 9 Consider a family of systems $\dot{x}=f(x, u, p)$ with a vector of parameters $p$ taking values from a compact set $\mathcal{P}$. Let the systems $\dot{x}=f(x, u, p)$ satisfy Assumptions A1-A4 for every $p$ in $\mathcal{P}$. Assume there exist parameter values $a, b$ in $\mathcal{P}$ such that the systems $\dot{x}=f(x, u, a)$ and $\dot{x}=f(x, u, b)$ are monotone on $\mathcal{D}_{M} \times \mathcal{U}_{\infty}$, where $\mathcal{D}_{M}=\cup_{q \in \mathcal{P}} \mathcal{D}_{f(\cdot, \cdot, q)}$ and

$$
f(x, u, a) \preceq_{x} f(x, u, p) \preceq_{x} f(x, u, b),
$$

for all $(x, u, p) \in \mathcal{D}_{M} \times \mathcal{U} \times \mathcal{P}$. Let also

$$
\begin{gathered}
s_{f(\cdot, \cdot, p)}^{0} \in \operatorname{int}\left(\bigcap_{q \in \mathcal{P}} \mathcal{A}\left(s_{f(\cdot, \cdot, q)}^{0}\right)\right), \\
s_{f(\cdot, \cdot, p)}^{1} \notin\left\{z \mid s_{f(\cdot, \cdot, a)}^{0} \preceq_{x} z \preceq_{x} s_{f(\cdot, \cdot, b)}^{0}\right\},
\end{gathered}
$$

for all $p$ in $\mathcal{P}$. Then the following relation holds:

$$
\mathcal{S}_{f(\cdot, \cdot, a)}^{-} \supseteq \mathcal{S}_{f(\cdot, \cdot, p)}^{-} \supseteq \mathcal{S}_{f(\cdot, \cdot, b)}^{-} \quad \forall p \in \mathcal{P} .
$$

The proof follows by setting $g(x, u)=f(x, u, a)$ and $r(x, u)=f(x, u, b)$ and noting that the conditions in (12), (13) imply the conditions in (8), (9) in the premise of Theorem 7 .

Theorem 7 states that if the bounding systems (3), (4) can be found, the switching sets $\mathcal{S}_{g}^{-}, \mathcal{S}_{r}^{-}$can be estimated, thereby providing approximations on the switching set $\mathcal{S}_{f}^{-}$. In what follows we provide a procedure to find monotone bounding systems if the system (2) is near-monotone, meaning that by removing some interactions between the states the system becomes monotone (see [21] for the discussion on nearmonotone systems).
Let there exist a single interaction which is not compatible with monotonicity, and let this interaction be between the states $x_{i}$ and $x_{j}$. This happens if, for example, the $(i, j)$ th entry in the Jacobian $\left\{\frac{\partial f_{i}}{\partial x_{j}}\right\}_{i, j}$ is smaller or equal to zero. A monotone system can be obtained by replacing the variable $x_{j}$ with a constant in the function $f_{i}(x, u)$, which removes the interaction between the states $x_{i}$ and $x_{j}$. If the set $\mathcal{D}$ is bounded then clearly we can find $\bar{x}_{j}$ and $\underline{x}_{j}$ such that $\bar{x}_{j} \geq x_{j} \geq \underline{x}_{j}$ for all $x \in \mathcal{D}$. If the set $\mathcal{D}$ is not bounded, then we need to estimate the bounds on the intersection of $\mathcal{A}\left(s_{f}^{0}\right)$ and the reachability set starting at $s_{f}^{0}$ for all admissible pulses. Let $g_{k}=r_{k}=f_{k}$ for all $k \neq i$, $g_{i}(x, u)=\left.f_{i}(x, u)\right|_{x_{j}=\underline{x}_{j}}$, and $r_{i}(x, u)=\left.f_{i}(x, u)\right|_{x_{j}=\bar{x}_{j}}$. It is straightforward to show that $\dot{x}=g(x, u)$, and $\dot{x}=r(x, u)$ are monotone systems and their vector fields are bounding the vector field $f$ from below and above, respectively. Note that in order to apply Theorem 7 we still need to check if these bounding systems satisfy Assumptions A1-A4.

In the case of Corollary 9 , the procedure is quite similar. If the system $\dot{x}=f(x, u, p)$ is monotone for all parameter values $p$, then we can find $a, b$ if there exists a partial order in the parameter space. That is a relation $\preceq_{p}$ such that for parameter values $p_{1}$ and $p_{2}$ satisfying $p_{1} \preceq_{p} p_{2}$ we have that

$$
f\left(x, u, p_{1}\right) \preceq_{x} f\left(x, u, p_{2}\right) \forall x \in \mathcal{D}, u \in \mathcal{U} .
$$

If a partial order is found, the values $a$ and $b$ are computed as minimal and maximal elements of $\mathcal{P}$ in the partial order $\preceq_{p}$. This idea is equivalent to treating parameters $p$ as inputs and showing that the system $\dot{x}=f(x, u, p)$ is monotone with respect to inputs $u$ and $p$.

\section{Computation of the Switching Separatrix}

The theoretical results in Section 3 guarantee the existence of the switching separatrix for monotone systems, but in order to compute $\mu(\tau)$ we resort to numerical algorithms.

Given a pair $(\mu, \tau)$ we can check if this pair is switching the system using simulations (that is, numerically integrating the corresponding differential equation). If the curve $\mu(\tau)$ is a monotone function, then for every $\tau$ there exists a unique pulse magnitude $\mu=\mu(\tau)$. Let $\mathcal{T}=\left\{\tau_{i}\right\}_{i=1}^{N}$ be such that $\tau_{\min }=\tau_{1} \leq \tau_{i} \leq \tau_{i+1} \leq \tau_{N}=\tau_{\max }$ for all $i$. Clearly, for every $\tau_{i}$ we can compute the corresponding $\mu_{i}$ using bisection. We start the algorithm by computing the value $\mu_{1}$ corresponding to $\tau_{1}$. Due to monotonicity of the switching separatrix, the minimal switching magnitude $\mu_{2}$ for the pulse length $\tau_{2}$ is smaller or equal to $\mu_{1}$. Therefore, we can save some computational effort by setting the upper bound on the computation of $\mu_{2}$ equal to $\mu_{1}$. The computation of the pairs $(\mu, \tau)$ can be parallelised by setting the same upper bound on $\mu_{i}, \cdots, \mu_{i+N_{\text {par }}}$, where $N_{\text {par }}$ is the number of independent computations. As an output we obtain $\mathcal{M}_{\text {min }}$ and $\mathcal{M}_{\max }$, which are the sets of pairs $(\mu, \tau)$ approximating the switching separatrix from below and above, respectively. 


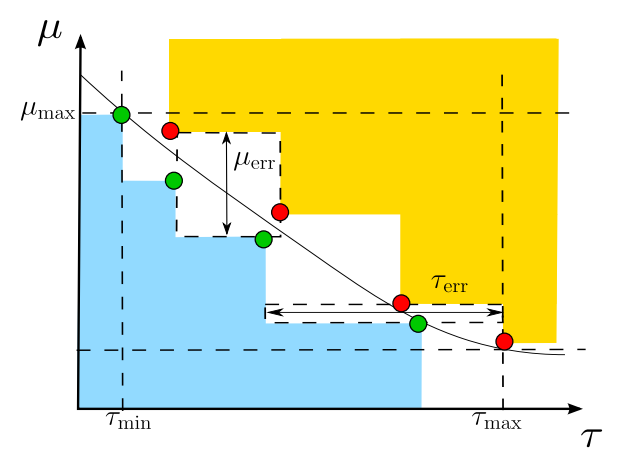

Fig. 4. Illustration of the error of computation of the switching separatrix between the values $\tau_{\min }, \tau_{\max }$. The black curve is the switching separatrix to be computed, the red and green circles are the upper and lower bounding points, respectively. The switching separatrix should lie between the coloured regions due to its monotonicity. The values $\mu_{\mathrm{err}}$ and $\tau_{\mathrm{err}}$ are the largest height and width of boxes inscribed between the coloured regions, respectively.

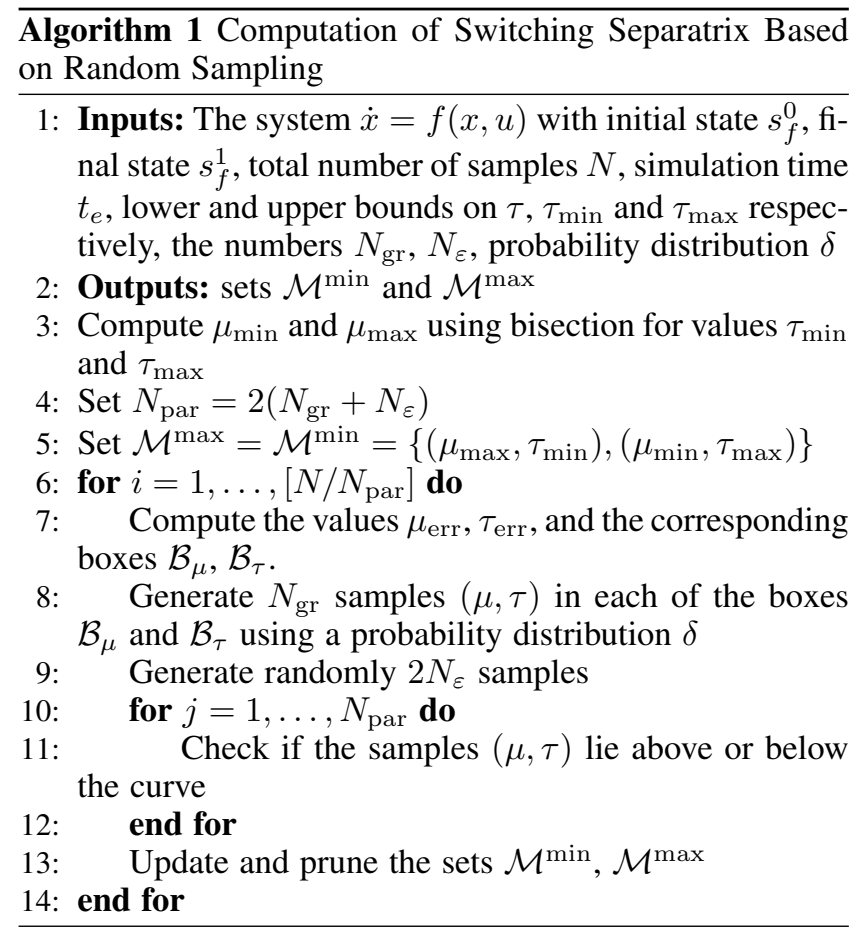

In order to evaluate the error of computing the switching separatrix consider Figure 4. According to the definitions in the caption of Figure 4 we define the relative error of the approximation as

$$
E_{\mathrm{rel}}=\left(\mu_{\mathrm{err}} /\left(\mu_{\max }-\mu_{\min }\right)+\tau_{\mathrm{err}} /\left(\tau_{\max }-\tau_{\min }\right)\right) / 2 .
$$

Note that, even if the green and red circles lie very close to each other the relative error can be substantial. In numerical simulations we use a logarithmic grid for $\tau$, which yields a significantly lower relative error in comparison with an equidistant grid. This can be explained by an observation that in many numerical examples $\mu(\tau)$ appears to be an exponentially decreasing curve.
There are a few drawbacks in the bisection algorithm. Firstly, it requires a large number of samples. Secondly, the choice of the grid is not automatic, which implies that for switching separatrices with different geometry the relative error on the same grid may be drastically different. Finally, the algorithm relies on the assumption that $\mu(\tau)$ is a graph of a monotone function, which may not be true. In order to overcome these difficulties, we have derived Algorithm 1 based on random sampling, which converges faster than the bisection algorithm, has higher sample efficiency, does not require a predefined grid and the graph assumption. Some of the steps in Algorithm 1 require additional explanation:

Step 7. Find two boxes: the box $\mathcal{B}_{\mu}$ with the maximal height (denoted as $\mu_{\mathrm{err}}$ ) and the box $\mathcal{B}_{\tau}$ with the maximal width (denoted as $\tau_{\text {err }}$ ) that can be inscribed between the coloured regions as depicted in Figure 4.

Step 9. Generate $N_{\varepsilon}$ samples of $\tau$ using a probability distribution $\delta$ between $\tau_{\min }$ and $\tau_{\max }$. For every $\tau$ generate a value $\mu$ using a distribution $\delta$ such that it lies in the area between the coloured regions. Repeat this step by first generating $\mu$ between $\mu_{\min }$ and $\mu_{\max }$ using a distribution $\delta$, and then generating $\tau$ for every generated $\mu$ in the area between the coloured regions.

Step 13. First, we update the sets $\mathcal{M}^{\text {min }}, \mathcal{M}^{\text {max }}$ by adding the samples lying below and above the switching separatrix, respectively. Then if there exist two pairs $\left(\mu_{1}, \tau_{1}\right)$ and $\left(\mu_{2}, \tau_{2}\right)$ in the set $\mathcal{M}^{\min }$ (resp., $\mathcal{M}^{\max }$ ) such that $\mu_{1} \leq \mu_{2}$ and $\tau_{1} \leq \tau_{2}$, then delete the pair $\left(\mu_{1}, \tau_{1}\right)$ from the set $\overline{\mathcal{M}}^{\text {min }}$ (resp., the pair $\left(\mu_{2}, \tau_{2}\right)$ from the set $\mathcal{M}^{\text {max }}$ ).

Note that Step 11 is the most computationally expensive part of the algorithm and its computation is distributed into $N_{\text {par }}$ independent tasks. In our implementation, we chose $\delta$ as a Beta distribution with parameters 1 and 3 and adjust the support to a specific interval. Note that the set between the coloured regions is getting smaller with every generated sample, hence the relative error of Algorithm 1 is a nonincreasing function of the total number of samples. In fact, numerical experiments show that this function is on average exponentially decreasing. After the sets $\mathcal{M}^{\text {min }}$ and $\mathcal{M}^{\text {max }}$ are generated one can employ machine learning algorithms to build a closed form approximation of a switching separatrix (e.g., Sparse Bayesian Learning [28]; see also [31], [17] for efficient algorithms).

\section{Illustration of Results on the LacI-TetR Switch}

Evaluation of the Computational Algorithm. Here we compare the bisection algorithm and Algorithm 1 with different parameter values, as well as their distributed implementations on the LacI-TetR switch introduced in Subsection 2.2. Note that Algorithm 1 does not depend explicitly on the dynamics of the underlying system, but depends only on the generated pairs $(\mu, \tau)$. Therefore, the convergence and sample efficiency results presented here will be valid for a broad 


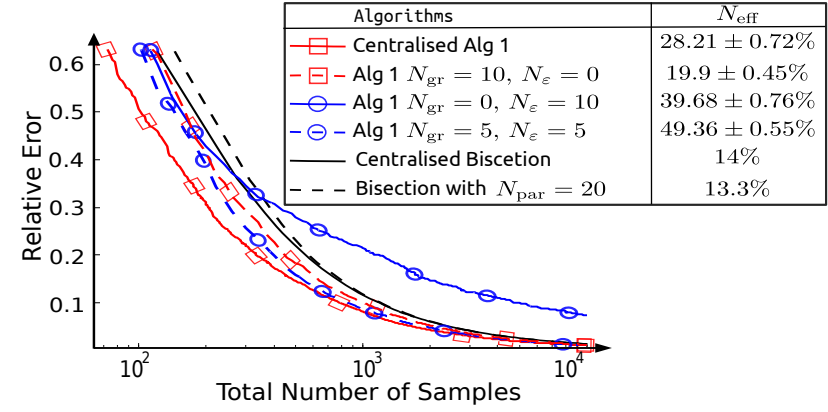

Fig. 5. Average error against total number of generated samples. The curves corresponding to the bisection algorithm are computed by a single run of the algorithm. The curves corresponding to $N_{\varepsilon}=0$ are averages over ten runs of Algorithm 1, while the curves for $N_{\varepsilon}>0$ are the averages over twenty runs of Algorithm 1 . Recall that $N_{\mathrm{par}}=2\left(N_{\mathrm{gr}}+N_{\varepsilon}\right)$ for Algorithm 1. In the table we list sample efficiency $N_{\text {eff }}$ in percent. In the notation $x \pm y, x$ stands for the emperical mean, and $y$ for the emperical standard deviation.

class of systems. In Figure 5, we compare the error against the total number of generated samples. Since checking if a sample lies above or below the curve is the most expensive part of both algorithms, the total number of samples reflects the computational complexity. In the case of Algorithm 1 with $N_{\varepsilon}=0$ the randomisation level is not high, hence an average over ten runs is sufficient to demonstrate the average behaviour of this algorithm. Note that Algorithm 1 with $N_{\varepsilon}=0$ outperforms the bisection algorithm in the centralised and parallelised settings.

Some computational effort in Algorithm 1 goes into computing the error. However, this effort appears to be negligible in comparison with numerically solving a differential equation for a given pair $(\mu, \tau)$ even for such an easy system as the toggle switch. We run the simulations on a computer equipped with Intel Core i7-4500U processor and 8GB of RAM. Using the centralised version of Algorithm 1 we achieved on average a relative error equal to 0.0448 in 87.65 seconds, while it took 89.17 seconds to obtain a relative error equal to 0.0842 with the bisection algorithm. For systems with a larger number of states the difference may be larger.

In Figure 5, we also compare the sample efficiency of the algorithms, which we define as

$$
N_{\text {eff }}=\left|\mathcal{M}^{\min } \cup \mathcal{M}^{\max }\right| / N
$$

where $N$ is the total number of generated samples, and $\left|\mathcal{M}^{\text {min }} \cup \mathcal{M}^{\text {max }}\right|$ is the number of samples in the set $\mathcal{M}^{\text {min }} \cup$ $\mathcal{M}^{\max }$. Results in Figure 5 indicate that Algorithm 1 has higher sample efficiency than the bisection algorithm.

Our results also indicate that Algorithm 1 with $N_{\mathrm{gr}}=5$, $N_{\varepsilon}=5$ has on average a higher empirical convergence rate and a higher sample efficiency than Algorithm 1 with $N_{\mathrm{gr}}=10, N_{\varepsilon}=0$. This indicates that a combination of nonzero $N_{\mathrm{gr}}, N_{\varepsilon}$ improves convergence and sample efficiency,
Switching separatrices

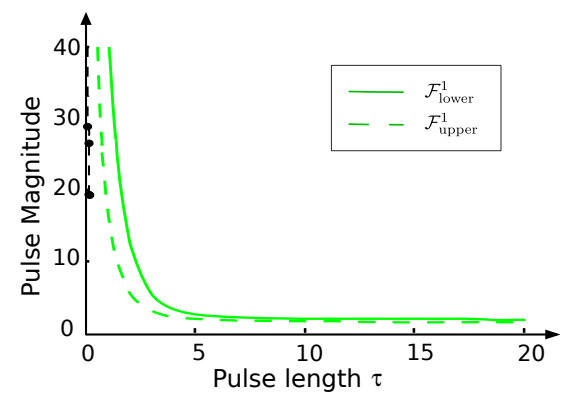

Fig. 6. Switching separatrices for the LacI-TetR system (5).

which can be explained as follows. When the total number of generated samples is low, we do not have sufficient information on the behaviour of the switching separatrix. Therefore we need to explore this behaviour by randomly generating samples, before we start minimising the relative error. This idea is similar to the so-called exploration/exploitation trade-off in reinforcement learning [27].

Robust Switching in the LacI-TetR System. We specify a system $\mathcal{F}_{\text {upper }}$ with $p_{1}=40, p_{4}=0.05, p_{6}=30, p_{9}=$ 0.1 and a system $\mathcal{F}_{\text {lower }}$ with $p_{1}=20, p_{4}=0.01, p_{6}=$ $45, p_{9}=0.3$. The remaining parameters are the same as in (6). After that we compute the switching separatrices and plot them in Figure 6. According to Corollary 9, the system with parameter values $p_{1} \in[20,40], p_{4} \in[0.01,0.005]$, $p_{6} \in[30,65], p_{9} \in[0.1,0.3]$ and the remaining parameters as in (6) will have switching separatrix lying between the solid and dashed green curves in Figure 6. This is quite remarkable considering the level of parameter variations. If other parameters are varied as well then the bounds on the sepratrices may be looser as discussed in [23]. Therein we also illustrate the application of Theorem 7 to a perturbed nonmonotone LacI-TetR switch.

\section{Further Counterexamples and Applications}

Toxin-Antitoxin System describes interaction between the toxin proteins $T$ and antitoxin proteins $A$ [4]:

$$
\begin{aligned}
\dot{T} & =\frac{\sigma_{T}}{\left(1+\frac{\left[A_{f}\right]\left[T_{f}\right]}{K_{0}}\right)\left(1+\beta_{M}\left[T_{f}\right]\right)}-\frac{1}{\left(1+\beta_{C}\left[T_{f}\right]\right)} T \\
\dot{A} & =\frac{\sigma_{A}}{\left(1+\frac{\left[A_{f}\right]\left[T_{f}\right]}{K_{0}}\right)\left(1+\beta_{M}\left[T_{f}\right]\right)}-\Gamma_{A} A+u \\
\varepsilon\left[\dot{A}_{f}\right] & =A-\left(\left[A_{f}\right]+\frac{\left[A_{f}\right]\left[T_{f}\right]}{K_{T}}+\frac{\left[A_{f}\right]\left[T_{f}\right]^{2}}{K_{T} K_{T T}}\right) \\
\varepsilon\left[\dot{T}_{f}\right] & =T-\left(\left[T_{f}\right]+\frac{\left[A_{f}\right]\left[T_{f}\right]}{K_{T}}+2 \frac{\left[A_{f}\right]\left[T_{f}\right]^{2}}{K_{T} K_{T T}}\right),
\end{aligned}
$$

where $\left[A_{f}\right],\left[T_{f}\right]$ is the number of free toxin and antitoxin proteins. In [4], the authors considered the model with $\varepsilon=0$. In order to simplify our analysis we set $\varepsilon=10^{-6}$. If the parameters are chosen as follows: $\sigma_{T}=166.28, K_{0}=$ 
$1, \beta_{M}=\beta_{c}=0.16, \sigma_{A}=10^{2}, \Gamma_{A}=0.2, K_{T}=K_{T T}=$ 0.3 , the system is bistable with two stable nodes, but is not monotone and we were not able to find bounding systems satisfying Assumptions A1-A4. Nevertheless, we approximated the switching sets and the switching separatrix on a mesh grid and noticed that the switching separatrix appears to be monotone. We can provide some intuition behind this phenomenon. With $\varepsilon$ tending to zero, we can apply singular perturbation theory (cf. [11]) to eliminate the states $\left[A_{f}\right]$, $\left[T_{f}\right]$. Numerical computations indicate that the reduced order system is not monotone in $\mathbb{R}_{>0}^{2}$, however, it is monotone around the stable equilibria, which may explain the monotonicity of the switching separatrix.

\section{Switching in a Mass Action Kinetics System from [30]:}

$$
\begin{aligned}
& \dot{x}_{1}=f_{1}\left(x_{1}, x_{2}\right)=2 k_{1} x_{2}-k_{2} x_{1}^{2}-k_{3} x_{1} x_{2}-k_{4} x_{1}+\beta u, \\
& \dot{x}_{2}=f_{2}\left(x_{1}, x_{2}\right)=k_{2} x_{1}^{2}-k_{1} x_{2} .
\end{aligned}
$$

We can assume without loss of generality that $k_{2}=1$, since we can remove one of the parameters using a simple change of variables. The unforced system has stable nodes $s^{0}, s^{1}$, and a saddle $s^{u}$ :

$s^{0}=\left(\begin{array}{l}0 \\ 0\end{array}\right) s^{u}=\left(\begin{array}{c}\frac{k_{1}-\sqrt{k_{1} D}}{2 k_{3}} \\ \left(\frac{\sqrt{k_{1}}-\sqrt{D}}{2 k_{3}}\right)^{2}\end{array}\right) s^{1}=\left(\begin{array}{c}\frac{k_{1}+\sqrt{k_{1} D}}{2 k_{3}} \\ \left(\frac{\sqrt{k_{1}}+\sqrt{D}}{2 k_{3}}\right)^{2}\end{array}\right)$

where $D=k_{1}-4 k_{3} k_{4}$. Naturally the system is bistable if $D>0$, globally asymptotically stable if $D<0$ and a saddle-node bifurcation occurs if $D=0$. It can be verified that the system is monotone on $\mathcal{D}=\left\{x_{1}, x_{2} \mid 0 \leq x_{1} \leq\right.$ $\left.2 k_{1} / k_{3}\right\}$, which also contains the equilibria and hence the system satisfies our assumptions.

The derivatives of $f_{1}, f_{2}$ with respect to $k_{1}$ do not have the same sign hence the system is not monotone with respect to parameter $k_{1}$. This term appears due to so-called mass action kinetics, which are common in biological applications and hence this problem is met often. A straightforward solution is to treat every instance of $k_{1}$ as an independent parameter. Hence we have a vector of parameters $\left[k_{11}, k_{3}, k_{4}, k_{12}\right]$, where $k_{11}$ is the instance of $k_{1}$ entering the first equation, and $k_{12}$ is the instance of $k_{1}$ entering the second equation. Let $k_{1} \in[7.7,8.3], k_{3} \in[1,1.2]$, $k_{4} \in[1,1.2]$ and consider the lower bounding parameter vector $p_{l}=[7.7,1.2,1.2,8.3]$, and the upper bounding parameter vector $p_{u}=[8.3,1,1,7.7]$. We apply Corollary 9 only to relatively small perturbations in parameters, since with larger variations the system becomes mono- or unstable. There is no indication that this problem is unique to this system, and does not appear in other mass-action systems.

We conclude this example by performing a sweep for the parameter $k_{1} \in[6,10]$, while $k_{2}=k_{3}=1$. Numerical simulations suggest that for any $k_{1} \in(6,10)$ the switching separatrix appears to lie between the blue and the red curves, which are switching separatrices for $k_{1}=6$ and $k_{1}=10$, respectively. Again we can only provide some intuition behind this observation. It is straightforward to verify that the gradient of $s^{u}$ with respect to $k_{1}$ is a negative vector, and the gradient of $s^{1}$ with respect to $k_{1}$ is a positive vector. Hence the equilibria depend on $k_{1}$ in the way which is consistent with a behaviour of a monotone system. This example may indicate that the behaviour of the equilibria is one of the necessary conditions allowing the switching separatrix to be a monotone curve and change monotonically with respect to parameter variations.

Shaping Pulses to Induce Oscillations in an Eight Species Generalised Repressilator. An eight species generalised repressilator is an academic example, where each of the species represses another species in a ring topology. The corresponding dynamic equations for a symmetric generalised repressilator are as follows:

$$
\begin{aligned}
& \dot{x}_{1}=\frac{p_{1}}{1+\left(x_{8} / p_{2}\right)^{p_{3}}}+p_{4}-p_{5} x_{1}+u_{1}, \\
& \dot{x}_{2}=\frac{p_{1}}{1+\left(x_{1} / p_{2}\right)^{p_{3}}}+p_{4}-p_{5} x_{2}+u_{2}, \\
& \dot{x}_{i}=\frac{p_{1}}{1+\left(x_{i-1} / p_{2}\right)^{p_{3}}}+p_{4}-p_{5} x_{i}, \forall i=3, \ldots 8,
\end{aligned}
$$

where $p_{1}=40, p_{2}=1, p_{3}=3, p_{4}=0.5$, and $p_{5}=1$. This system has two stable equilibria $s^{1}$ and $s^{2}$ and is monotone with the respect to $P_{x} \mathbb{R}^{n} \times P_{u} \mathbb{R}^{2}$, where $P_{x}=\operatorname{diag}([1,-$ $1,1,-1,1,-1,1,-1]), P_{u}=\operatorname{diag}([1,-1])$. It can actually be shown that the system is strongly monotone in the interior of $\mathbb{R}_{\geq 0}^{8}$ for all positive parameter values. The control signal $u_{1}$ can switch the system from the state $s^{1}$ to the state $s^{2}$, while the control signal $u_{2}$ can switch the system from the state $s^{2}$ to the state $s^{1}$. The switching separatrix for the control signal $u_{1}$ is depicted in the upper panel of Figure 7. Note that the separatrix is identical for the control signal $u_{2}$, since the repressilator is symmetric.

Numerical simulations suggest that the trajectories exhibit an oscillatory behaviour, while switching between the stable steady states using a pulse. This is in agreement with previous studies that showed the existence of unstable periodic orbits [26] in a generalised repressilator. Switching trajectories of species $x_{1}$ for various pairs $(\mu, \tau)$ are depicted in the lower panel of Figure 7. The observations made in the caption of Figure 7 indicate that the closer the pair $(\mu, \tau)$ is to the switching separatrix the longer oscillations will persist.

We can set up another control problem: to induce oscillations in the generalised repressilator. One can address the problem by forcing the trajectories to be close to the unstable periodic orbit of the system, which, however, is very hard to compute. In [24], it was proposed to track other periodic trajectories instead. However, the solution was very computationally expensive and offering little insight into the problem. Here we will use pulses to induce oscillations as was proposed in [26]. In contrast to [26], we provide a way to shape all possible pulses inducing oscillations. 

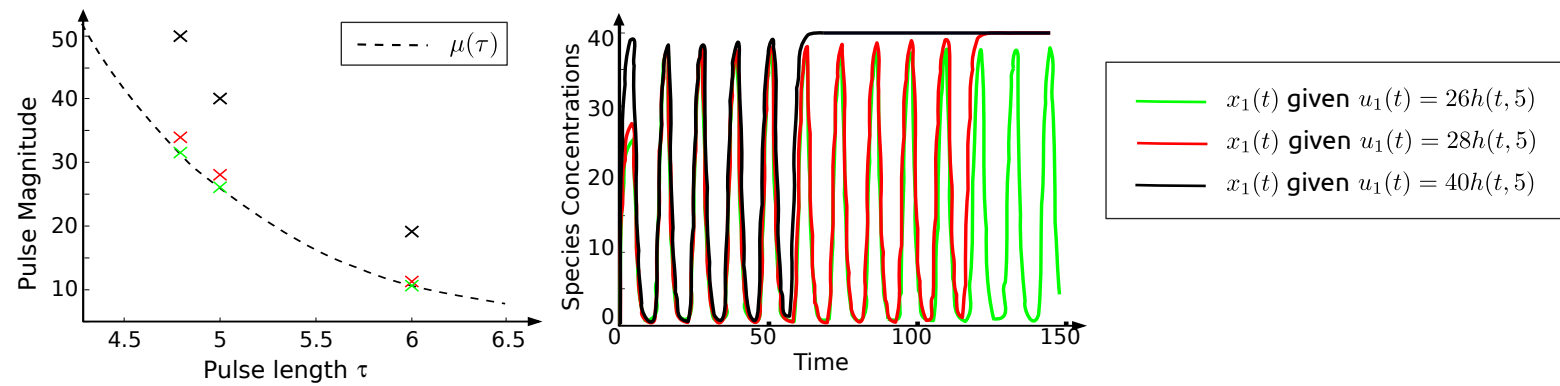

Fig. 7. Switching between steady states in a generalised repressilator system. All trajectories generated by the pairs $(\mu, \tau)$ corresponding to the black crosses in the left panel will converge to a steady state with the same rate as the black curve in the right panel. Similar correspondence is valid for the red and green crosses in the left panel and the red and green curves in the right panel. This observation indicates that the closer the pair $(\mu, \tau)$ is to the switching separatrix the longer oscillations will persist.

Let the initial point be $s^{1}$. We can shape the control signal $u_{1}$ to switch to the state $s^{2}$. Once we have reached the state $s^{2}$, we can shape the control signal $u_{2}$ to switch back to the state $s^{1}$ and so on. During switching we will observe oscillations depending on the position of the pair $(\mu, \tau)$ with respect to the switching separatrix. Now we need to define an automatic way of switching between the steady states. Let $\mathcal{M}$ be equal to $\left\{z \mid s^{1} \preceq_{x} z \preceq_{x} s^{2}\right\}$. It can be verified that the unstable equilibrium lies in $\mathcal{M}$, which typically holds for monotone systems. Moreover, the trajectories observed in Figure 7 lie in $\mathcal{M}$ due to monotonicity. Let $\varepsilon>0$ and $\mathcal{M}_{\varepsilon}=\left\{z \mid s^{1}+\varepsilon P_{x} \mathbf{1} \preceq_{x} z \preceq_{x} s^{2}-\varepsilon P_{x} \mathbf{1}\right\}$, where $\mathbf{1}$ is the vector of ones. Clearly $\mathcal{M}_{\varepsilon} \subset \mathcal{M}$ and if $\varepsilon$ is small enough then oscillating trajectories lie in $\mathcal{M}_{\varepsilon}$. Since the repressilator is symmetric we can assume that the shape of pulses for both $u_{1}$ and $u_{2}$ is the same. In this case we can formalise our control strategy as follows. If the event $x\left(t_{e}\right) \preceq_{x} s^{1}+\varepsilon P_{x} \mathbf{1}$ occurs at time $t_{e}$, then

$$
u_{1}(\cdot)=\mu h\left(\cdot, t_{e}+\tau\right) \quad u_{2}(\cdot)=0
$$

If the event $x\left(t_{e}\right) \succeq_{x} s^{2}-\varepsilon P_{x} \mathbf{1}$ occurs at time $t_{e}$, then

$$
u_{1}(\cdot)=0 \quad u_{2}(\cdot)=\mu h\left(\cdot, t_{e}+\tau\right)
$$

Note that we change the entire control signals when the event occurs, which we assume happens at some time $t_{e}$. Due to

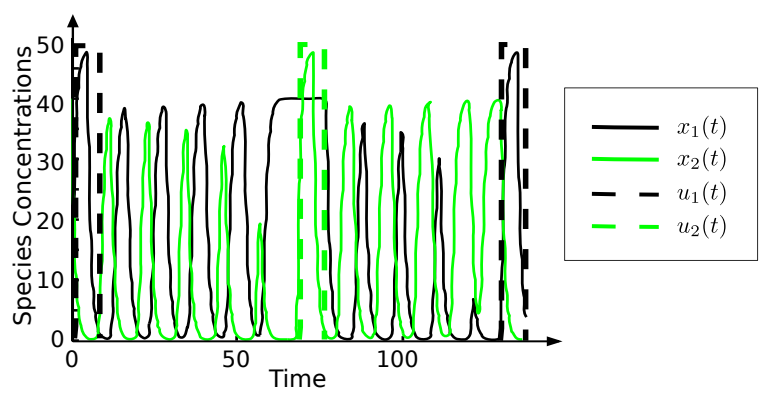

Fig. 8. Inducing oscillatory behaviour in the generalised repressilator system with eight species. The pulses for both $u_{1}$ and $u_{2}$ are equal, and are generated using a pair $(\mu, \tau)=(48,4.8)$. The pair $(48,4.8)$ lies relatively far from the switching separatrix, hence the time between switches is not large. this fact, the pulse $\mu h\left(\cdot, t_{e}+\tau\right)$ is of length $\tau$. The resulting trajectories for the species $x_{1}$ and $x_{2}$, as well as control signals are depicted in Figure 8. Our control algorithm falls into the class of event-based control, with the events occurring if $x\left(t_{e}\right)$ leaves $\mathcal{M}_{\varepsilon}$. For any small enough $\varepsilon$, our control strategy will induce oscillations. However, it is not clear how to compute an $\varepsilon$ for which $\mathcal{M}_{\epsilon}$ is much smaller than $\mathcal{M}$ but also contains the oscillating trajectories.

\section{Conclusion and Discussion}

In this paper we have presented a framework for shaping pulses to control bistable systems. Our main motivation comes from control problems arising in Synthetic Biology, but the results hold in other classes of bistable systems. We considered the problem of switching between stable steady states using temporal pulses. We showed that the problem is feasible, if the flow of the controlled system can be bounded from above and below by flows of monotone systems. We presented a detailed analysis of the conditions needed for switching, together with an algorithm to compute the pulse's length and duration. We illustrated the theory with a number of case studies and counterexamples that shed light on the limitations of the approach and highlight the need for further theoretical tools to control bistable non-monotone systems.

Throughout this work we did not take into account stochasticity in the model dynamics, which can be particularly important in biochemical systems [6]. Noisy bistable dynamics can be controlled, for example, using reinforcement learning algorithms as the ones described in [24,25]. These approaches, however, require large amounts of measurement data that are typically impractical to acquire. A promising extension to our results is the switching problem in stochastic bimodal systems. This requires the use of the socalled stochastically monotone Markov decision processes, for which a whole new set of theoretical tools needs to be developed. Work in this direction started in [22] and the references within, addressing the extension of the concept of monotonicity to stochastic systems. 


\section{References}

[1] BM Adams, HT Banks, Hee-Dae Kwon, and Hien T Tran. Dynamic multidrug therapies for HIV: Optimal and STI control approaches. Mathematical Biosciences and Engineering, 1(2):223-241, 2004.

[2] D. Angeli and E.D. Sontag. Monotone control systems. IEEE Trans. Autom. Control, 48(10):1684-1698, 2003.

[3] Jennifer AN Brophy and Christopher A Voigt. Principles of genetic circuit design. Nature methods, 11(5):508-520, 2014.

[4] Ilaria Cataudella, Kim Sneppen, Kenn Gerdes, and Namiko Mitarai. Conditional cooperativity of toxin-antitoxin regulation can mediate bistability between growth and dormancy. PLoS computational biology, 9(8):e1003174, 2013.

[5] Luigi Chisci and Paola Falugi. Asymptotic tracking for constrained monotone systems. IEEE Tran Automatic Control, 51(5):873-879, 2006.

[6] M.B. Elowitz, A.J. Levine, E.D. Siggia, and P.S. Swain. Stochastic gene expression in a single cell. Science Signalling, 297(5584):1183, 2002.

[7] T. Gardner, C. R. Cantor, and J. J. Collins. Construction of a genetic toggle switch in escherichia coli. Nature, 403:339-342, 2000.

[8] M. Gennat and B. Tibken. Computing guaranteed bounds for uncertain cooperative and monotone nonlinear systems. In Proc. IFAC World Congress, pages 4846-4851, 2008.

[9] J. Jiang, X. Liang, and X.-Q. Zhao. Saddle-point behavior for monotone semiflows and reaction-diffusion models. J. Differential Equations, 203(2):313-330, 2004.

[10] Ahmad S Khalil and James J Collins. Synthetic biology: applications come of age. Nature Reviews Genetics, 11(5):367-379, 2010.

[11] Hassan K. Khalil. Nonlinear systems. Prentice Hall, 2002.

[12] A. Levskaya, O. D. Weiner, W. A. Lim, and C. A. Voigt. Spatiotemporal control of cell signalling using a light-switchable protein interaction. Nature, 461:997-1001, 2009.

[13] F. Menolascina, M. Di Bernardo, and D. Di Bernardo. Analysis, design and implementation of a novel scheme for in-vivo control of synthetic gene regulatory networks. Automatica, Special Issue on Systems Biology, 47(6):1265-1270, April 2011.

[14] J. T. Mettetal, D. Muzzey, C. Gomez-Uribe, and A. van Oudenaarden. The Frequency Dependence of Osmo-Adaptation in Saccharomyces cerevisiae. Science, 319(5862):482-484, 2008.

[15] P.-J. Meyer, A. Girard, and E. Witrant. Controllability and invariance of monotone systems for robust ventilation automation in buildings. In Proc. Conf, Decision Control, pages 1289-1294. IEEE, 2013.

[16] A. Milias-Argeitis, S. Summers, J. Stewart-Ornstein, I. Zuleta, D. Pincus, H. El-Samad, M. Khammash, and J. Lygeros. In silico feedback for in vivo regulation of a gene expression circuit. Nature biotechnology, 2011.

[17] W. Pan, A. Sootla, and G.-B. Stan. Distributed reconstruction of nonlinear networks: An ADMM approach. In Preprints IFAC World Congress, Aug 2014.

[18] Priscilla E M Purnick and Ron Weiss. The second wave of synthetic biology: from modules to systems. Nat. Rev. Mol. Cell Biol., 10(6):410-422, jun 2009.

[19] N. Ramdani, N. Meslem, and Y. Candau. Computing reachable sets for uncertain nonlinear monotone systems. Nonlinear Analysis: Hybrid Systems, 4(2):263-278, 2010.

[20] H.L. Smith. Monotone dynamical systems: an introduction to the theory of competitive and cooperative systems, volume 41. American Mathematical Soc., 2008.

[21] E D Sontag. Monotone and near-monotone biochemical networks. Systems and Synthetic Biology, 1(2):59-87, 2007.
[22] A. Sootla. On monotonicity and propagation of order properties. In Proc. American Control Conf., pages 3144-3149, Chicago, Il, July 2015.

[23] A. Sootla, D. Oyarzún, D. Angeli, and G.-B. Stan. Shaping pulses to control bistable biological systems. In Proc Amer Control Conf, pages 3138-3143, 2015.

[24] A. Sootla, N. Strelkowa, D. Ernst, M. Barahona, and G.-B. Stan. On reference tracking using reinforcement learning with application to gene regulatory networks. In Conf. Decision Control, pages 40864091, Florence, Italy, Dec. 10-13 2013.

[25] A. Sootla, N. Strelkowa, D. Ernst, M. Barahona, and G.-B. Stan. Toggling the genetic switch using reinforcement learning. In Proc 9th French Meeting on Planning, Decision Making and Learning, Liège, Belgium, May 2014.

[26] N. Strelkowa and M. Barahona. Switchable genetic oscillator operating in quasi-stable mode. J R Soc Interface, 7(48):1071-1082, 2010.

[27] R. S. Sutton and A. G. Barto. Reinforcement Learning: An Introduction (Adaptive Computation and Machine Learning). The MIT Press, March 1998

[28] M. E. Tipping. Sparse bayesian learning and the relevance vector machine. J. Machine Learning Research, 1:211-244, 2001.

[29] J. Uhlendorf, A. Miermont, T. Delaveau, G. Charvin, F. Fages, S. Bottani, G. Batt, and P. Hersen. Long-term model predictive control of gene expression at the population and single-cell levels. Proc. Nat. Academy Sciences, 109(35):14271-14276, 2012.

[30] T. Wilhelm. The smallest chemical reaction system with bistability. BMC systems biology, 3(1):90, 2009.

[31] D. Wipf, S. Nagarajan, J. Platt, D. Koller, Y. Singer, and S. Roweis. A new view of automatic relevance determination. Advances in Neural Information Processing Systems, 20:1625-1632, 2008.

[32] J. W. Zwolak, J. J. Tyson, and L. T. Watson. Finding all steady state solutions of chemical kinetic models. Nonlinear analysis: real world applications, 5(5):801-814, 2004.

\section{A Proofs}

Proof of Proposition 3. (1) Here we simply need to notice that by monotonicity with $t \rightarrow \infty$ we have

$$
\xi(\mu) \leftarrow \phi_{g}\left(t, s_{g}^{0}, \mu\right) \preceq_{x} \phi_{g}\left(t, s_{g}^{0}, \lambda\right) \rightarrow \xi(\lambda) .
$$

Similarly we can show that $\eta(\mu) \preceq_{x} \eta(\lambda)$.

(2) First, we need to show that $\xi(\mu) \in \mathcal{A}\left(s_{g}^{0}\right)$ for all $0<$ $\mu<\mu_{\min }$. This is straightforward, since due the definition of $\mu_{\text {min }}$ the flow $\phi_{g}\left(t, s_{g}^{0}, \mu h(\cdot, \tau)\right)$ converges to $s_{g}^{0}$ for all the pairs $(\mu, \tau) \in \mathcal{S}^{-}$for $0<\mu<\mu_{\min }$. Hence the limit $\lim _{t \rightarrow \infty} \phi_{g}\left(t, s_{g}^{0}, \mu\right)$ belongs to $\mathcal{A}\left(s_{g}^{0}\right)$.

Now, we show that $s_{g}^{0} \prec_{x} s_{q}^{1}$. Consider $u=0$ and $v=$ $\lambda h(\cdot, \tau)$ such that $(\lambda, \tau) \in \mathcal{S}^{+}$. Therefore we have

$$
s_{g}^{0}=\phi_{g}\left(t, s_{g}^{0}, u\right) \preceq_{x} \phi_{g}\left(t, s_{g}^{0}, v\right) \rightarrow s_{g}^{1},
$$

with $t \rightarrow \infty$. Since $s_{g}^{0}$ is not equal to $s_{g}^{1}$, we have $s_{g}^{0} \prec_{x} s_{g}^{1}$. Now the claim $\xi(\mu) \prec_{x} \eta(\mu)$ for all $0<\mu<\mu_{\min }$ follows by monotonicity.

(3) Consider $\mu=\mu_{\min }+\varepsilon$ and $\tau$ large enough that the 
pair $(\mu, \tau) \in \mathcal{S}^{+}$. Hence the flow $\phi_{g}\left(t, s_{g}^{0}, \mu h(\cdot, \tau)\right)$ converges to $s_{g}^{1}$. By monotonicity we have that $\phi_{g}\left(t, s_{g}^{0}, \mu\right) \succeq_{x}$ $\phi_{g}\left(t, s_{g}^{0}, \mu h(\cdot, \tau)\right)$, which implies that $\xi(\mu) \succeq_{x} s_{g}^{1}$ for arbitrarily small $\varepsilon>0$. Since $\xi\left(\mu_{\text {min }}-\varepsilon\right)$ lies in $\mathcal{A}\left(s_{g}^{0}\right)$ we have that $\left\|\xi\left(\mu_{\min }-\varepsilon\right)-\xi(\mu)\right\|_{2}>0$, which proves the claim.

Proof of Theorem 4 . 1) $\Rightarrow$ 2) A. It is straightforward to verify that the premise of Theorem 4 implies that any point lying in the set $\mathcal{S}_{f}^{-}$is path-wise connected to a point in the neighbourhood of the origin. In order to show that the set is simply connected, it is left to prove that there are no holes in the set $\mathcal{S}_{f}^{-}$. Let $\eta(\mu, \tau)$ be a closed curve which lies in $\mathcal{S}_{f}^{-}$. Consider the set

$\mathcal{S}^{\eta}=\left\{(\mu, \tau) \mid 0<\mu \leq \mu^{\eta}, 0<\tau \leq \tau^{\eta},\left(\mu^{\eta}, \tau^{\eta}\right) \in \eta(\mu, \tau)\right\}$.

Since the set $\mathcal{S}_{f}^{-}$is in $\mathbb{R}_{>0}^{2}$, the set $\mathcal{S}^{\eta}$ contains the set enclosed by the curve $\eta(\mu, \tau)$. It is straightforward to show that $\mathcal{S}^{\eta}$ is a subset of $\mathcal{S}_{f}^{-}$by the premise of the theorem. Hence there are no holes in the area enclosed by the arbitrary curve $\eta \in \mathcal{S}_{f}^{-}$. Since the curve $\eta$ is in $\mathbb{R}^{2}$ we can shrink this curve continuously to a point, which belongs to the set $\mathcal{S}_{f}^{-}$. Since the curve is an arbitrary closed curve in $\mathcal{S}_{f}^{-}$, the set $\mathcal{S}_{f}^{-}$is simply connected.

B. Let us show here that there exists a set of maximal elements in $\mathcal{S}_{f}^{-}$. Let a pair $\left(\mu^{u}, \tau^{u}\right)$ not belong to $\mathcal{S}_{f}^{-}$. If there exists a pair $(\mu, \tau) \in \mathcal{S}_{f}^{-}$such that $\mu \geq \mu^{u}, \tau \geq \tau^{u}$, then by the arguments above the pair $\left(\mu^{u}, \tau^{u}\right)$ must also belong to $\mathcal{S}_{f}^{-}$. Hence, all pairs $(\mu, \tau)$ such that $\mu \geq \mu^{u}, \tau \geq \tau^{u}$ do not belong to $\mathcal{S}_{f}^{-}$. This implies that there exists a set of maximal elements of $\mathcal{S}_{f}^{-}$in the standard partial order, which is a segment of the boundary of $\mathcal{S}_{f}^{-}$excluding the points with $\mu$ and $\tau$ equal to zero.

C. It is left to establish that the set of maximal elements is unordered. Let the mapping $\mu_{f}(\tau)$ denote the set of maximal elements of $\mathcal{S}_{f}^{-}$and let $\tau_{1}<\tau_{2}$. Since the mapping $\mu_{f}(\tau)$ are the maximal elements in $\mathcal{S}_{f}^{-}$, we cannot have $\mu_{f}\left(\tau_{1}\right)<\mu_{f}\left(\tau_{2}\right)$. Hence, $\mu_{f}\left(\tau_{1}\right) \geq \mu_{f}\left(\tau_{2}\right)$, for all $\tau_{1}<\tau_{2}$. 2) $\Rightarrow$ 1) The claim follows directly from the fact that there exists a set of maximal elements $\mu_{f}(\tau)$ in the simply connected set $\mathcal{S}_{f}^{-}$.

Proof of Theorem 6. 1) Due to Assumption A4, there exists at least one point $\left(\mu^{l}, \tau^{l}\right)$ in $\mathcal{S}_{g}^{-}$. Let us show that if a pair $\left(\mu^{l}, \tau^{l}\right)$ belongs to $\mathcal{S}_{g}^{-}$, then all pairs $(\mu, \tau)$ such that $0<$ $\mu \leq \mu^{l}, 0<\tau \leq \tau^{l}$ also belong to $\mathcal{S}_{g}^{-}$. By the definition of the order in $u$, for every $0<\mu \leq \mu^{l}, 0<\tau \leq \tau^{l}$ we have $0 \preceq_{u} \mu h(t, \tau) \preceq_{u} \mu^{l} h\left(t, \tau^{l}\right)$. The following then holds

$$
s_{g}^{0} \preceq_{x} \phi_{g}\left(t ; s_{g}^{0}, \mu h(\cdot, \tau)\right) \preceq_{x} \phi_{g}\left(t ; s_{g}^{0}, \mu^{l} h\left(\cdot, \tau^{l}\right)\right) .
$$

By assumption, there exists a $T$ such that for all $t>T$ the flow $\phi_{g}\left(t ; s_{g}^{0}, \mu^{l} h\left(\cdot, \tau^{l}\right)\right)$ belongs to $\mathcal{A}\left(s_{g}^{0}\right)$ and converges

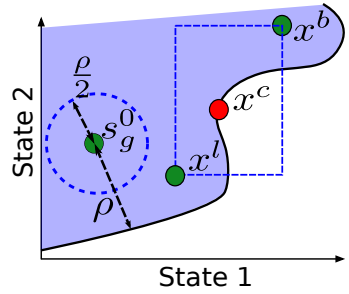

Fig. A.1. An illustration to the proof of Lemma 10 for a two-state system. We assume that $x^{b}, x^{l}$ lie in $\mathcal{A}\left(s_{g}^{0}\right)$ (violet area) and $x^{b} \succeq_{x} x^{c} \succeq_{x} x^{l}$ with $x^{c}$ lying on the boundary of $\partial \mathcal{A}\left(s_{g}^{0}\right)$. We show that, if the trajectory $\phi_{g}\left(t, x^{c}, 0\right)$ is on the boundary of $\mathcal{A}\left(s_{g}^{0}\right)$, it has to converge to $s_{g}^{0}$, which cannot be true due to monotonicity of the system.

to $s_{g}^{0}$. Therefore $\phi_{g}\left(t ; s_{g}^{0}, \mu h(\cdot, \tau)\right)$ converges to $s_{g}^{0}$ with $t \rightarrow+\infty$, and consequently the pair $(\mu, \tau)$ does not toggle the system and thus belongs to $\mathcal{S}_{g}^{-}$. Therefore, by Theorem 4 $\mu_{g}\left(\tau_{1}\right) \geq \mu_{g}\left(\tau_{2}\right)$, for all $\tau_{1}<\tau_{2}$.

2) Due to Assumption A4, there exists at least one point $\left(\mu^{l}, \tau^{l}\right)$ in $\mathcal{S}_{g}^{+}$. Similarly to point $\left.\mathbf{1}\right)$ above, we can show that, if a pair $\left(\mu^{l}, \tau^{l}\right)$ belongs to $\mathcal{S}_{g}^{+}$, then by continuity of solutions to (3) there exist $\bar{\varepsilon}>0, \bar{\delta}>0$ such that the pairs $(\mu+\varepsilon, \tau+\delta)$ also belong to $\mathcal{S}_{g}^{+}$for all $0<\varepsilon<\bar{\varepsilon}, 0<\delta<\bar{\delta}$. Hence the set $\mathcal{S}_{g}^{+}$has a non-empty interior. The rest of the proof is the same as the proof of the implication 1) $\Rightarrow 2$ ) in Theorem 4.

3) We prove the result by contradiction. Let there exist a $\tau$ and an interval $I=\left(\mu_{1}, \mu_{2}\right)$ such that for all $\mu \in$ $I$ the flow $\phi_{g}\left(t, s_{g}^{0}, \mu h(\cdot, \tau)\right)$ does not converge to $s_{g}^{0}$ or $s_{g}^{1}$, but belongs to the interior of $\mathcal{D}_{g}$. This means that the flow $\phi_{g}\left(t, s_{g}^{0}, \mu h(\cdot, \tau)\right)$ evolves on the separatrix $\partial \mathcal{A}$ between domains of attraction $\mathcal{A}\left(s_{g}^{0}\right)$ and $\mathcal{A}\left(s_{g}^{1}\right)$ for all $t>\tau$. Let $\mu_{1}, \mu_{2}$ belong to $I$ and $\mu_{1}<\mu_{2}$, which implies that $\phi_{g}\left(t, s_{g}^{0}, \mu_{1} h(\cdot, \tau)\right) \ll_{x} \phi\left(t, s_{g}^{0}, \mu_{2} h(\cdot, \tau)\right)$ and both flows belong to $\partial \mathcal{A}$. This in turn implies that the set $\partial \mathcal{A}$ contains comparable points, that is, the set $\partial \mathcal{A}$ is not unordered. We arrive at a contradiction, and hence the interval $I$ is empty and for any $\tau$ there exists a unique $\mu_{g}(\tau)$. This is equivalent to $\mu_{g}(\cdot)$ being a graph of a function. Using similar arguments, we can show that the inverse mapping $\mu_{g}^{-1}(\tau)$ is also a graph of a function, which indicates that $\mu_{g}(\cdot)$ is a decreasing function.

Similarly, we can show that for any $\mu$ the minimum value of $\tau_{2}-\tau_{1}$, such that the pairs $\left(\mu, \tau_{1}-\varepsilon\right) \in \mathcal{S}_{g}^{-},\left(\mu, \tau_{2}+\varepsilon\right) \in \mathcal{S}_{g}^{+}$ $\forall \varepsilon>0$, is zero. This readily implies that $\mu_{g}(\tau)=\nu_{g}(\tau)$ and completes the proof.

Before we proceed with the proof of Theorem 7 we will need two additional results.

Lemma 10 Let the system $\dot{x}=g(x, 0)$ satisfy Assumption $A 1$ and be monotone on $\mathcal{A}\left(s_{g}^{0}\right)$, where $s_{g}^{0}$ is a stable steady state and $\mathcal{A}\left(s_{g}^{0}\right)$ is its domain of attraction. Let $x^{b}$ and $x^{l}$ belong to $\mathcal{A}\left(s_{g}^{0}\right)$. Then all points $z$ such that $x^{l} \preceq_{x} z \preceq_{x} x^{b}$ belong to $\mathcal{A}\left(s_{g}^{0}\right)$. 
Proof. We will show the result by contradiction. Let $x^{l}$, $x^{b}$ belong to $\mathcal{A}\left(s_{g}^{0}\right)$, let $x^{c}$ be such that $x^{l} \preceq_{x} x^{c} \preceq_{x} x^{b}$ and not belong to $\mathcal{A}\left(s_{g}^{0}\right)$. Without loss of generality assume that $x^{c}$ belongs to the boundary of $\mathcal{A}\left(s_{g}^{0}\right)$ (see Figure A.1). Therefore the flow $\phi_{g}\left(t, x^{c}, 0\right)$ is on the boundary of $\mathcal{A}\left(s_{g}^{0}\right)$. Let the distance between $s_{g}^{0}$ and this boundary be equal to $\rho$. Clearly there exists a time $T_{1}$ such that for all $t>T_{1}$ the following inequalities hold

$$
\begin{gathered}
\left\|s_{g}^{0}-\phi_{g}\left(t, x^{b}, 0\right)\right\|_{2}<\rho / 2 \\
\left\|s_{g}^{0}-\phi_{g}\left(t, x^{l}, 0\right)\right\|_{2}<\rho / 2 .
\end{gathered}
$$

Moreover, there exists a time $T_{2}>T_{1}$ such that for all $t>T_{2}$ and all $z$ such that $\phi_{g}\left(t, x^{l}, 0\right) \preceq_{x} z \preceq_{x} \phi_{g}\left(t, x^{b}, 0\right)$ we have $\left\|s_{g}^{0}-z\right\|_{2}<\rho / 2$. Now build a sequence $\left\{x^{n}\right\}_{n=1}^{\infty}$ converging to $x^{c}$ such that all $x^{n}$ lie in $\mathcal{A}\left(s_{g}^{0}\right)$ and $x^{l} \preceq_{x}$ $x^{n} \preceq_{x} x^{b}$. Due to monotonicity on $\mathcal{A}\left(s_{g}^{0}\right)$, we have

$$
\phi_{g}\left(t, x^{l}, 0\right) \preceq_{x} \phi_{g}\left(t, x^{n}, 0\right) \preceq_{x} \phi_{g}\left(t, x^{b}, 0\right)
$$

for all $n$ and $t$. Hence, for all $t>T_{2}$, we also have that $\left\|s_{g}^{0}-\phi_{g}\left(t, x^{n}, 0\right)\right\|_{2}<\rho / 2$. Since the sequence $\left\{x^{n}\right\}_{n=1}^{\infty}$ converges to $x^{c}$, by continuity of solutions to (3), for all $t>T_{2}$ we have $\left\|s_{g}^{0}-\phi_{g}\left(t, x^{c}, 0\right)\right\|_{2} \leq \rho / 2$, which is a contradiction since $\left\|s_{g}^{0}-\phi_{g}\left(t, x^{c}, 0\right)\right\|_{2} \geq \rho$ for all $t$.

Lemma 11 Consider the dynamical systems $\dot{x}=f(x, u)$ and $\dot{x}=g(x, u)$ satisfying Assumption Al. Let one of the systems be monotone on $\mathcal{D}_{M} \times \mathcal{U}_{\infty}$. If $g(x, u) \succeq_{x} f(x, u)$ for all $(x, u) \in \mathcal{D}_{M} \times \mathcal{U}$ then for all $t$, and for all $x_{2} \succeq_{x} x_{1}$, $u_{2} \succeq_{u} u_{1}$ we have $\phi_{g}\left(t ; x_{2}, u_{2}\right) \succeq_{x} \phi_{f}\left(t ; x_{1}, u_{1}\right)$.

Proof. Without loss of generality let $\dot{x}=g(x, u)$ be monotone with respect to $\mathbb{R}_{\geq 0}^{n}$. Let $\mathbf{1}$ be a vector of ones, $x_{2}^{m}=$ $x_{2}+1 / m \cdot \mathbf{1}$, and $\dot{x}=g(x, u)+\mathbf{1} / m$. Denote the flow of this system $\phi^{m}\left(t ; x_{2}^{m}, u_{2}\right)$. Clearly for a sufficiently small $t$ the condition $\phi_{m}\left(t ; x_{2}^{m}, u_{2}\right) \gg_{x} \phi_{f}\left(t ; x_{1}, u_{1}\right)$ holds. Assume there exists a time $s$, for which this condition is violated. That means that for some $i$ we have $\phi_{m}^{i}\left(t ; x_{2}^{m}, u_{2}\right)>$ $\phi_{f}^{i}\left(t ; x_{1}, u_{1}\right)$ for all $0 \leq t<s$, where the superscript $i$ denotes an $i$-th element of the vector. While at time $s$ we have $\phi_{m}^{i}\left(s ; x_{2}^{m}, u_{2}\right)=\phi_{f}^{i}\left(s ; x_{1}, u_{1}\right)$. Hence we conclude that

$$
\left.\frac{d}{d t}\left(\phi_{m}^{i}\left(t ; x_{2}^{m}, u_{2}\right)-\phi_{f}^{i}\left(t ; x_{1}, u_{1}\right)\right)\right|_{t=s} \leq 0
$$

However,

$$
\begin{gathered}
\left.\frac{d \phi_{f}^{i}\left(t ; x_{1}, u_{1}\right)}{d t}\right|_{t=s}=f_{i}\left(\phi_{f}\left(s ; x_{1}, u_{1}\right), u_{1}\right)< \\
g_{i}\left(\phi_{f}\left(s ; x_{1}, u_{1}\right), u_{1}\right)+1 / m \leq \\
g_{i}\left(\phi_{m}\left(s ; x_{2}^{m}, u_{2}\right), u_{2}\right)+1 / m=\left.\frac{d \phi_{m}^{i}\left(t ; x_{2}^{m}, u_{2}\right)}{d t}\right|_{t=s} .
\end{gathered}
$$

The inequality in (A.2) holds due to the bound $g(x, u)+$ $\mathbf{1} / m \succ_{x} f(x, u)$. Since the system $\dot{x}=g(x, u)+\mathbf{1} / m$ is monotone, the inequality in (A.3) holds as well according to the remark after Proposition 2. This chain of inequalities contradicts (A.1), hence for all $t$ we have that $\phi_{m}\left(t ; x_{2}^{m}, u_{2}\right) \gg_{x} \phi_{f}\left(t ; x_{1}, u_{1}\right)$. With $m \rightarrow+\infty$, by continuity of solutions we obtain $\phi_{g}\left(t, x_{2}, u_{2}\right) \succeq_{x} \phi_{f}\left(t ; x_{1}, u_{1}\right)$, which completes the proof.

Proof of Theorem 7. A. First we note that the assumption in (8) implies that $s_{g}^{0} \preceq_{x} s_{f}^{0} \preceq_{x} s_{r}^{0}$. Indeed, take $x_{0}$ from the interior of the intersection of the sets $\mathcal{A}\left(s_{g}^{0}\right), \mathcal{A}\left(s_{f}^{0}\right)$, $\mathcal{A}\left(s_{r}^{0}\right)$. By Lemma 11 for all $t$, we have $\phi_{g}\left(t, x_{0}, 0\right) \preceq_{x}$ $\phi_{f}\left(t, x_{0}, 0\right) \preceq_{x} \phi_{r}\left(t, x_{0}, 0\right)$, and thus taking the limit $t \rightarrow$ $\infty$ we get $s_{g}^{0} \preceq_{x} s_{f}^{0} \preceq_{x} s_{r}^{0}$.

B. Next we show that $g(x, u) \preceq_{x} f(x, u)$ for all $(x, u) \in$ $\mathcal{D}_{M} \times \mathcal{U}$ implies that $\mathcal{S}_{g}^{-} \supseteq \mathcal{S}_{f}^{-}$. Let the set $\mathcal{V}$ be such that $u=\mu h(\cdot, \tau) \in \mathcal{V}$ if $(\mu, \tau) \in \mathcal{S}_{f}^{-}$.

Due to $s_{g}^{0} \preceq_{x} s_{f}^{0}$ and $g \preceq_{x} f$ on $\mathcal{D}_{M} \times \mathcal{U}_{\infty}$, by Lemma 11 , we have that $s_{g}^{0} \preceq_{x} \phi_{g}\left(t ; s_{g}^{0}, u\right) \preceq_{x} \phi_{f}\left(t ; s_{f}^{0}, u\right)$, for all $u \in \mathcal{V}$. Note that the first inequality is due to monotonicity of the system $\dot{x}=g(x, u)$. The flow $\phi_{f}\left(t ; s_{f}^{0}, u\right)$ converges to $s_{f}^{0}$ with $t \rightarrow+\infty$. Therefore, there exists a time $T$ such that for all $t>T$ we have $s_{g}^{0} \preceq_{x} \phi_{g}\left(t ; s_{g}^{0}, u\right) \ll_{x} s_{f}^{0}+\varepsilon \mathbf{1}$ for some positive $\varepsilon$. Moreover, we can pick an $\varepsilon$ such that $s_{f}^{0}+\varepsilon \mathbf{1}$ lies in $\mathcal{A}\left(s_{g}^{0}\right)$ (due to (8)). Since the system $\dot{x}=g(x, u)$ is monotone, according to Lemma 10, the flow $\phi_{g}\left(t ; s_{g}^{0}, u\right)$ lies in $\mathcal{A}\left(s_{g}^{0}\right)$. Hence, no $u$ in $\mathcal{V}$ toggles the system $\dot{x}=g(x, u)$ either and we conclude that $\mathcal{S}_{g}^{-} \supseteq \mathcal{S}_{f}^{-}$. The proof that $\mathcal{S}_{g}^{-} \supseteq$ $\mathcal{S}_{r}^{-}$follows using the same arguments as above.

C. Finally, we show that $\mathcal{S}_{f}^{-} \supseteq \mathcal{S}_{r}^{-}$. Let the set $\mathcal{W}$ be such that $u=\mu h(\cdot, \tau) \in \mathcal{W}$ if $(\mu, \tau) \in \mathcal{S}_{r}^{-}$.

Due to $s_{g}^{0} \preceq_{x} s_{f}^{0} \preceq_{x} s_{r}^{0}$ and $g \preceq_{x} f \preceq_{x} r$ on $\mathcal{D}_{M} \times \mathcal{U}_{\infty}$, by Lemma 11, we have that

$$
\phi_{g}\left(t ; s_{g}^{0}, u\right) \preceq_{x} \phi_{f}\left(t ; s_{f}^{0}, u\right) \preceq_{x} \phi_{r}\left(t ; s_{f}^{0}, u\right),
$$

for all $u \in \mathcal{W}$. Now, monotonicity of $\dot{x}=g(x, u)$ implies that $s_{g}^{0} \preceq_{x} \phi_{g}\left(t ; s_{g}^{0}, u\right)$. Furthermore, there exists a $T$ such that $s_{g}^{0} \preceq_{x} \phi_{f}\left(t ; s_{f}^{0}, u\right) \preceq_{x} s_{r}^{0}+\varepsilon \mathbf{1}$ for all $t>T$, for all $u \in \mathcal{W}$ and some small positive $\varepsilon$. This is due to the fact that $\phi_{r}\left(t ; s_{f}^{0}, u\right) \rightarrow s_{r}^{0}$ with $t \rightarrow+\infty$. We can also choose an $\varepsilon$ such that $s_{r}^{0}+\varepsilon \mathbf{1}$ lies in $\mathcal{D}_{M}$ due to (8). Hence, the flow of $\dot{x}=f(x, u)$ for all $u \in \mathcal{W}$ belongs to the set $\left\{z \mid s_{g}^{0} \preceq_{x} z \preceq_{x} s_{r}^{0}+\varepsilon \mathbf{1}\right\}$ for all $t>T$.

Now, assume there exists $u^{c} \in \mathcal{W}$ that toggles the system $\dot{x}=f(x, u)$. This implies that the flow $\phi_{f}\left(t ; s_{f}^{0}, u^{c}\right)$ converges to $s_{f}^{1}$ with $t \rightarrow \infty$. Therefore we have that $s_{f}^{1}$ belongs to the set $\left\{z \mid s_{g}^{0} \preceq_{x} z \preceq_{x} s_{r}^{0}+\varepsilon \mathbf{1}\right\}$ for an arbitrarily small $\varepsilon$, and consequently $s_{f}^{1} \preceq_{x} s_{r}^{0}$. This contradicts the condition (9) in the premise of Theorem 7. Hence, no $u$ in $\mathcal{W}$ toggles the system $\dot{x}=f(x, u)$ and $\mathcal{S}_{f}^{-} \supseteq \mathcal{S}_{r}^{-}$. 